\title{
On the Mechanism of $\alpha \mathrm{C}$ Polymer Formation in Fibrin
}

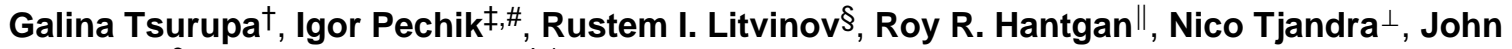 \\ W. Weisel ${ }^{\S}$, and Leonid Medved ${ }^{\dagger},{ }^{*}$ \\ tCenter for Vascular and Inflammatory Diseases and the Department of Biochemistry, University \\ of Maryland School of Medicine, Baltimore, Maryland 21201, United States \\ ¥Walter Reed Army Institute of Research, Silver Spring, Maryland 20910, United States \\ §Department of Cell and Developmental Biology, Perelman School of Medicine at the University \\ of Pennsylvania, Philadelphia, Pennsylvania 19104, United States \\ "Department of Biochemistry, Wake Forest University School of Medicine, Winston-Salem, North \\ Carolina 27157, United States \\ ${ }^{\perp}$ Laboratory of Molecular Biophysics, National Heart, Lung, and Blood Institute, National Institutes \\ of Health, Bethesda, Maryland 20892, United States
}

\section{Abstract}

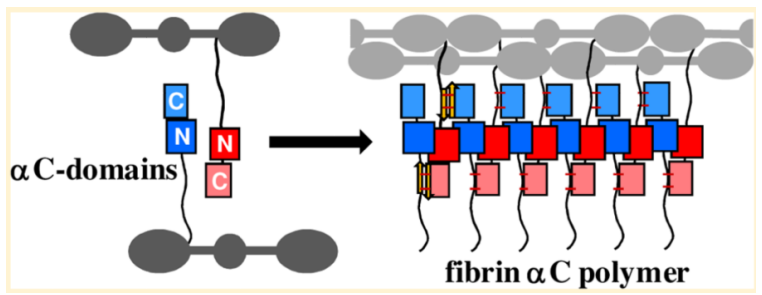

Our previous studies revealed that the fibrinogen aC-domains undergo conformational changes and adopt a physiologically active conformation upon their self-association into aC polymers in fibrin. In the present study, we analyzed the mechanism of $\mathrm{aC}$ polymer formation and tested our hypothesis that self-association of the $\mathrm{aC}$-domains occurs through the interaction between their $\mathrm{N}$ terminal subdomains and may include $\beta$-hairpin swapping. Our binding experiments performed by size-exclusion chromatography and optical trap-based force spectroscopy revealed that the aCdomains self-associate exclusively through their $\mathrm{N}$-terminal subdomains, while their $\mathrm{C}$-terminal subdomains were found to interact with the $\mathrm{aC}$-connectors that tether the $\mathrm{aC}$-domains to the bulk of the molecule. This interaction should reinforce the structure of aC polymers and provide the proper orientation of their reactive residues for efficient cross-linking by factor XIIIa. Molecular modeling of self-association of the $\mathrm{N}$-terminal subdomains confirmed that the hypothesized $\beta$ hairpin swapping does not impose any steric hindrance. To "freeze" the conformation of the $\mathrm{N}$ terminal subdomain and prevent the hypothesized $\beta$-hairpin swapping, we introduced by sitedirected mutagenesis an extra disulfide bond between two $\beta$-hairpins of the bovine Aa406-483 fragment corresponding to this subdomain. The experiments performed by circular dichroism revealed that Aa406-483 mutant containing Lys429Cys/Thr463Cys mutations preserved its $\beta$ -

(C) 2012 American Chemical Society

*Corresponding Author. lmedved@ som.umaryland.edu; phone: (410) 706-8065; fax: (410) 706-8121.

\#Present Address

Venenum Biodesign, Hamilton, NJ 08691-1810.

Notes

The authors declare no competing financial interest. 
sheet structure. However, in contrast to wild-type Aa406-483, this mutant had lower tendency for oligomerization, and its structure was not stabilized upon oligomerization, in agreement with the above hypothesis. On the basis of the results obtained and our previous findings, we propose a model of fibrin aC polymer structure and molecular mechanism of assembly.

Fibrinogen aC-domains mediate fibrin(ogen) activity in various physiological and pathological processes. By interacting with each other, they promote lateral aggregation of protofibrils during fibrin assembly 1,2 and play an important role in determining the structure and properties of fibrin clots and their susceptibility to fibrinolysis. ${ }^{3}$ The aC-domains interact with factor XIII, ${ }^{4}$ which covalently cross-links them upon activation thereby reinforcing fibrin clots, and control activation of this factor. ${ }^{5}$ In addition, they modulate fibrinolysis by interacting with plasminogen and its activator, tPA, and inhibitor, $\mathrm{a}_{2^{-}}$ antiplasmin. ${ }^{6-8}$ They promote cell adhesion and spreading through the interaction of their Aa572-574 RGD sequence with cell surface integrins ${ }^{9,10}$ and may be involved in modulation of fibrin-dependent angiogenesis. ${ }^{11}$ The aC-domains may also contribute to the development of atherothrombosis through their interaction with apo(a) component of lipoprotein(a). ${ }^{12}$

Each of the two aC-domains of the fibrinogen molecule is formed by the C-terminal portion of the Aa chain (amino acid residues Aa392-610) and is tethered to the bulk of the molecule with a flexible aC-connector (residues Aa221-391); together they comprise a structure called the aC region. ${ }^{12,13}$ The aC-domain contains a compact structure whose presence was established by differential scanning calorimetry ${ }^{14-16}$ and electron microscopy. ${ }^{17-19}$ Recent study revealed that each aC-domain consists of two independently folded units, $\mathrm{N}$ - and C-terminal subdomains formed by the Aa392-503 and Aa504-610 residues, respectively. ${ }^{20}$ The NMR solution structure of the recombinant Aa406-483 fragment corresponding to the $\mathrm{N}$-terminal subdomain of the bovine fibrinogen $\mathrm{aC}$-domain revealed that this fragment contains two $\beta$-hairpins which form a mixed four-stranded parallel/antiparallel $\beta$-sheet. ${ }^{21,22}$ The recombinant Aa425-503 fragment corresponding to the $\mathrm{N}$-terminal subdomain of the human fibrinogen $\mathrm{aC}$-domain was shown to have a similar fold. ${ }^{20}$ Thus, the three-dimensional structure of the $\mathrm{N}$-terminal subdomain of the aC-domain has been established, but that of its $\mathrm{C}$-terminal subdomain remains to be defined.

Numerous studies suggest that in fibrinogen two aC-domains interact with each other and with the central $\mathrm{E}$ region of the molecule, whereas upon conversion of fibrinogen into fibrin they dissociate and switch from intra- to intermolecular interaction, thus promoting lateral aggregation of protofibrils. ${ }^{1-3,15,19,23,24}$ Such intermolecular interaction between the aCdomains in fibrin results in formation of aC polymers whose structure is reinforced by cross-linking with activated factor XIII (factor XIIIa). Other studies revealed that the aCdomains are inert in fibrinogen; however, in fibrin they become reactive and interact with a number of proteins including plasminogen, tPA, $a_{2}$-antiplasmin, and apolipoprotein(a). ${ }^{6,8,12}$ Altogether, these studies suggest that conversion of fibrinogen into fibrin is accompanied by conformational changes in the aC-domains, resulting in the exposure or formation of their multiple binding sites. A recent study confirmed that the aC-domains adopt a physiologically active conformation upon self-association (polymerization) and partially clarified the structure of these domains in aC polymers. ${ }^{25}$ However, the mechanism of such self-association, as well as the detailed structure of aC polymers, is still unclear.

The ability of the isolated aC-domains to self-associate into soluble oligomers was demonstrated in the studies with the recombinant fragments corresponding to human and bovine aC-domains and their $\mathrm{N}$-terminal subdomains. ${ }^{20,22}$ This process was shown to be concentration-dependent and reversible, suggesting that it mimics self-association (polymerization) of the tethered aC-domains in fibrin. ${ }^{20,25}$ It was also found that the 
thermodynamic stability of the monomeric forms of these fragments is low while that of their oligomeric forms is significantly increased. ${ }^{20,22}$ On the basis of these and some other findings, we hypothesized that self-association of the aC-domains into aC polymers in fibrin occurs through the interaction between their $\mathrm{N}$-terminal subdomains and may include $\beta$-hairpin swapping. ${ }^{20,22}$ However, this hypothesis does not take into account the recently discovered interaction between the aC-domain and aC-connector, ${ }^{24}$ which may also contribute to aC polymer formation. It is also unclear whether the C-terminal subdomains contribute to self-association of the aC-domains. The major goal of the present study was to test this hypothesis and to further clarify the mechanism of self-association of the aCdomains and the structure of fibrin aC polymers.

\section{EXPERIMENTAL PROCEDURES}

\section{Preparation of the Recombinant $\alpha C$-Domain Fragments}

Recombinant Aa221-391 and Aa392-610 fragments corresponding to the human fibrinogen aC-connector and aC-domain, respectively, were prepared as described earlier. ${ }^{16}$ Recombinant Aa392-503 and Aa504-610 fragments corresponding to the $\mathrm{N}$ - and Cterminal subdomains of the human fibrinogen aC-domain, respectively, were expressed in $E$. coli and subsequently purified and refolded by the recently described procedures. ${ }^{8}$ Recombinant Aa406-483 fragment corresponding to the truncated variants of the Nterminal subdomain of the bovine fibrinogen aC-domain was prepared as described earlier. $^{22}$

\section{Preparation of Aa406-483 Fragment Mutants}

The Thr428Cys/Thr463Cys, Lys429Cys/Thr463Cys, and Val426Cys/His461Cys double mutants of the bovine Aa406-483 fragment were produced by site-directed mutagenesis using QuikChange Multi Site-Directed Mutagenesis kit (Stratagene). The pET-20b construct containing DNA encoding the Aa406-483 fragment ${ }^{22}$ was modified by using the following mutagenic primers: $5^{\prime}-$

GTCGTTCATGCTCTAAAGTCATTTGCAAGACAGTTACAAATGCCGACG-3' and 5' CGTCGGCATTTGTAACTGTCTTGCAAATGACTTTAGAGCATGAACGAC-3'

(forward and reverse primers, respectively, for Thr428Cys mutation); $5^{\prime}$ GTCGTTCATGCTCTAAAGTCATTACATGCACAGTTACAAATGCCGACGG-3' and 5' -CCGTCGGCATTTGTAACTGTGCATGTAATGACTTTAGAGCATGAACGAC-3' (forward and reverse primers, respectively, for Lys429Cys mutation); $5^{\prime}$ GGACTTCGACTGGCACCATTGCTTTCCTTCGAGGGGTAACC-3' and 5' GGTTACCCCTCGAAGGAAAGCAATGGTGCCAGTCGAAGTCC-3' (forward and reverse primers, respectively, for Thr463Cys mutation); $5^{\prime}$ CCACTCGTCGTTCATGCTCTAAATGCATTACAAAGACAGTTACAAATG-3' and 5' CATTTGTAACTGTCTTTGTAATGCATTTAGAGCATGAACGACGAGTGG-3' (forward and reverse primers, respectively, for Val426Cys mutation); $5^{\prime}$ GACGCGGACTTCGACTGGTGCCATACTTTTCCTTCGAG-3' and 5' CTCGAAGGAAAAGTATGGCACCAGTCGAAGTCCGCGTC-3' (forward and reverse primers, respectively, for His461Cys mutation). These primers introduced the desired mutations, which were confirmed by sequence analysis.

All expressed mutants were found in inclusion bodies, from which they were purified by the procedure described earlier for preparation of the wild-type Aa406-483 fragment. ${ }^{22}$ The purified mutants were refolded by slow dialysis from urea at $4{ }^{\circ} \mathrm{C}$ using the previously described protocol ${ }^{16}$ and further purified by size-exclusion chromatography performed on a Superdex S-75 column equilibrated with TBS (20 mM Tris buffer, pH 7.4, with $150 \mathrm{mM}$ $\mathrm{NaCl}$ ) and $0.2 \mathrm{mM} \mathrm{PMSF}$ at $4{ }^{\circ} \mathrm{C}$. The refolded mutants were concentrated to $\sim 1 \mathrm{mg} / \mathrm{mL}$ 
with a Centriprep 10 concentrator (Millipore), filtered through $0.2 \mu \mathrm{m}$ filter unit, and stored at $4{ }^{\circ} \mathrm{C}$.

\section{Preparation of Cross-Linked Fibrin and Its a Chain Polymers}

Cross-linked fibrin was prepared by incubation of $3 \mathrm{mg} / \mathrm{mL}$ human fibrinogen in $20 \mathrm{mM}$ HEPES buffer (pH 7.4) containing $150 \mathrm{mM} \mathrm{NaCl}$ and $10 \mathrm{mM} \mathrm{CaCl}_{2}$, with thrombin at 2 $\mathrm{NIH} \mathrm{u} / \mathrm{mL}$ and factor XIII at 24 Loewy $\mathrm{u} / \mathrm{mL}$, respectively; aprotinin at $400 \mathrm{u} / \mathrm{mL}$ was added to inhibit possible fibrinolytic activity. After $60 \mathrm{~h}$ of incubation at room temperature, crosslinked fibrin clot was transferred into a freshly prepared mixture of $6 \mathrm{M}$ guanidine- $\mathrm{HCl}, 50$ mM TCEP [tris(2-carboxyethyl)-phosphine hydrochloride] (Calbiochem), and $30 \mathrm{mM}$ NEM ( $N$-ethylmaleimide) (Sigma, St. Louis, MO); the latter two agents reduced fibrin's disulfide bonds to thiols and blocked them to prevent oxidation. Cross-linked fibrin was completely dissolved in this mixture upon incubation for $3 \mathrm{~h}$ at room temperature. The fraction corresponding to cross-linked a chain polymers was separated by size-exclusion chromatography on a Superdex 200 column equilibrated with 3 M guanidine-HCl. A mixture of fibrinogen chains with blocked thiols was prepared by incubation of fibrinogen in the same mixture.

\section{Protein Concentration Determination}

Concentrations of the recombinant Aa406-483 mutants and cross-linked fibrin a chain polymers were determined spectrophotometrically using the extinction coefficients calculated from the amino acid compositions. ${ }^{26}$ For all three mutants the calculated extinction coefficient $E_{280,1 \%}$ was 6.4 , and that for cross-linked fibrin a chain polymers was 10.1. Molecular masses of Thr428Cys/Thr463Cys, Lys429Cys/Thr463Cys, and Val426Cys/ His461Cys mutants, and fibrin a chain equal to 8964, 8937, 8930, and $64614 \mathrm{Da}$, respectively, were calculated based on their amino acid composition. ${ }^{26}$ Note that the values of molecular masses of the recombinant fragments take into account the $\mathrm{NH}_{2}$-terminal fMet residue (see above) while the numbering of these fragments does not. The molecular masses and $E_{280,1 \%}$ for the remaining recombinant fragments were determined previously. $8,16,20,22$

\section{Size-Exclusion Chromatography}

Analytical size-exclusion chromatography was used to analyze the aggregation state of the Aa406-483 fragment and its mutants. The experiments were performed with a fast protein liquid chromatography system (FPLC, Pharmacia) on a Superdex 75 column at flow rate of $1.0 \mathrm{~mL} / \mathrm{min}$ and $4{ }^{\circ} \mathrm{C}$. Typically, $50 \mu \mathrm{L}$ of Aa406-483 fragment or its mutants at different concentrations were loaded onto the column equilibrated with $20 \mathrm{mM}$ Tris buffer ( $\mathrm{pH} 7.4)$ containing 0.15 or $2 \mathrm{M} \mathrm{NaCl}$ and followed by elution with the same buffer. Protein elution was monitored by measuring absorbance at $280 \mathrm{~nm}$. To determine the molecular masses of monomeric and oligomeric forms of the recombinant fragments, the column was calibrated with a gel filtration LMW calibration kit (Amersham Biosciences).

\section{Optical Trap-Based Force Spectroscopy}

Optical trap-based rupture force spectroscopy previously applied to study bimolecular protein-protein interactions ${ }^{27-29}$ was used to precisely quantify interactions between surface-bound aC-domain fragments. One of the fragments was covalently attached to a latex bead that was trapped by a focused laser beam and repeatedly touched to a pedestal coated with the other reacting fragment. In the event of a bonding interaction between the fragments, the forces required to rupture the bond were measured and collected into $5 \mathrm{pN}$ wide bins. Only forces $>10 \mathrm{pN}$ were analyzed to avoid optical artifacts. The peak of the force histogram represents the strength of bonds, and the cumulative binding probability characterizes the reactivity of the aC-domain fragments. In order to make the measurements 
comparable with the previously published data on the interactions of other aC-domain fragments, ${ }^{24}$ the experimental conditions described in the earlier paper were scrupulously reproduced. The only variation was the increased surface density of the immobilized fragments to make the interactions more pronounced; this resulted in about 1.5 -fold higher binding probability. Importantly, the surface density of the aC-domain fragment and its Cand $\mathrm{N}$-terminal subdomain fragments in this study was kept similar due to equimolar amounts of the fragments used during immobilization performed under identical conditions.

\section{Circular Dichroism Study}

Circular dichroism (CD) measurements were made with a Jasco-810 spectropolarimeter. CD spectra of the wild-type Aa406-483 fragment and individual fractions of its mutant were recorded using a $0.01 \mathrm{~cm}$ path-length quartz cuvette at $4{ }^{\circ} \mathrm{C}$. Thermally induced unfolding curves were obtained by monitoring the ellipticity at $227 \mathrm{~nm}$ in $0.1 \mathrm{~cm}$ path-length quartz cuvette, while increasing the temperature at a rate of $1{ }^{\circ} \mathrm{C} / \mathrm{min}$ with a Peltier type PFD-425S attachment. The experiments were performed in $20 \mathrm{mM}$ Tris buffer, $\mathrm{pH} 7.4$, containing 0.15 or $2 \mathrm{M} \mathrm{NaCl}$. All CD data were expressed as the mean residue ellipticity, [ $\theta$ ], in units of degrees centimeter squared per decimole.

\section{Dynamic Light Scattering}

Dynamic light scattering (DLS) measurements ${ }^{30}$ were performed with a Malvern Instruments ZEN $1600^{31}$ to characterize particle size distributions with samples of solubilized cross-linked fibrin chains, cross-linked a chain polymers, and fibrinogen chains. Prior to DLS measurements, the samples were dialyzed overnight against a solution containing $3 \mathrm{M}$ guanidine- $\mathrm{HCl}$, and the measurements were performed in this solution. Data were collected in triplicate runs from each sample, with each run comprising 10-15 intensity autocorrelation functions. These data were analyzed by the CONTIN algorithm to obtain intensity-weighted, $z$-average size distributions. ${ }^{32}$ The resultant hydrodynamic diameters were corrected for the increased viscosity in guanidine- $\mathrm{HCl}$ solution. ${ }^{33,34}$ Molecular masses were estimated from the resultant hydrodynamic data using an empirical power law for random coil proteins which predicts $R_{\mathrm{h}}=M_{r}^{0.56}$, where $R_{\mathrm{h}}$ represents hydrodynamic radius and $M_{\mathrm{r}}$ represents molecular mass with $R_{\mathrm{h}}$ in nm and $M_{\mathrm{r}}$ in kDa. ${ }^{35,36}$

\section{Molecular Modeling}

Molecular modeling study of self-association of the N-terminal subdomain was conducted based on the average minimized NMR structure of the monomeric fragment Aa406-483 (PDB entry 2JOR). Prior to modeling oligomers, the topology of the second $\beta$-hairpin of Aa406-483 was adjusted to that of the ideal $\beta$-structure by manual alterations of the main chain dihedral angles on the computer graphics using Discovery Studio Visualizer 2.5 (Accelrys). The adjusted structure was then refined by conjugate gradient energy minimization with CNS. ${ }^{37}$ At the final stage of minimization the harmonic restraints applied initially to the main chain atoms were removed allowing to relieve possible tensions in the backbone.

The model of Aa406-483 oligomer assembled in a "side-by-side" manner was built manually on the computer graphics using the refined structure of monomers as building blocks. Modeling Aa406-483 oligomers reinforced by $\beta$-hairpin swapping required, however, substantial alterations to the tertiary structure of the monomeric unit. The alterations were performed manually upon modeling the arrangements of the monomers into dimers. The initial arrangements were then refined by short-term molecular dynamics with CNS running simulations at a constant temperature and using a harmonic force to restrain the mobility of main chain atoms. Next, the refined structures of the dimers were manually assembled into oligomers. The assembling process for all three models was guided by the 
topology of the $\beta$-sheet. The obtained models were subjected to the refinement by rigid body dynamics with X-PLOR. ${ }^{38}$ The final adjustment was done by a conjugate gradient energy minimization of individual atomic positions.

The initial residue candidates for the replacement with Cys aiming to introduce a disulfide linkage between the middle $\beta$-strands and prevent $\beta$-hairpin swapping were selected by comparative conformational analysis using the whole set of NMR structures available for the Aa406-483 fragment (PDB entry 2JOR). Then, in the average minimized structure of Aa406-483, side chains of the selected candidate residues were substituted with that of Cys and corresponding disulfide linkages were built manually utilizing computer graphics, followed by the adjustment with two steps energy minimization. At the first step, tight harmonic restrains were applied to main chain atoms of the substitutes and their closest neighbors while keeping the rest of the structure frozen. At the second step, the restraints were loosened helping the local polypeptide chain backbone to accommodate the new disulfide linkage.

\section{RESULTS}

\section{Testing Self-Association of the C-terminal Subdomain of the aC-Domain}

Formation of aC polymers in fibrin includes self-association of the aC-domains and subsequent covalent cross-linking of these domains to the aC-connectors by factor XIIIa. ${ }^{25}$ Our previous studies with the bovine Aa406-483 fragment and its human counterpart, Aa425-503 fragment, both corresponding to the truncated $\mathrm{N}$-terminal subdomain of the aC-domain, revealed that each of them forms oligomers (self-associate) in a concentrationdependent and reversible manner. ${ }^{20,22}$ This suggests that the homophilic interaction between the $\mathrm{N}$-terminal subdomains contributes to self-association of the aC-domains in aC polymers. To test whether such interaction may also occur between the Cterminal subdomains, we carried out experiments similar to those previously performed with the Nterminal subdomain fragments. ${ }^{20,22}$ Namely, the recombinant Aa504-610 fragment, corresponding to the $\mathrm{C}$-terminal subdomain of the human aC-domain, was dialyzed against TBS (20 mM Tris buffer, $\mathrm{pH} 7.4$, with $0.15 \mathrm{M} \mathrm{NaCl}$ ) and then concentrated to 3.2, 6.5, and $11.1 \mathrm{mg} / \mathrm{mL}(269,546$, and $932 \mu \mathrm{M}$, respectively). No pellet or turbidity increase at $350 \mathrm{~nm}$ was observed in all three samples analyzed immediately after the concentration. When the samples were then applied to a Superdex 75 column equilibrated with the same buffer, the fragment at all three concentrations eluted in a single peak corresponding to its monomeric form and no soluble oligomers similar to those formed by the N-terminal subdomain fragments ${ }^{20,22}$ were detected (not shown). Similar results were obtained when these samples were analyzed after overnight incubation. Thus, in contrast to the $\mathrm{N}$-terminal subdomain fragments, the $\mathrm{C}$-terminal subdomain fragment did not form any soluble oligomers even at a very high concentration.

To further test whether the C-terminal subdomains have a tendency to interact with each other (self-associate), we turned to the optical trap-based force spectroscopy, which we previously used to detect weak intermolecular interactions. ${ }^{24}$ In the experiments performed by this method, the Aa504-610 fragment was covalently immobilized to a latex bead that was trapped by a focused laser beam and touched to a motionless pedestal coated covalently with the same fragment. When the two surfaces coated with this fragment were touching repeatedly, the interactions formed an exponentially decreasing spectrum of rupture forces without any peaks and with a cumulative binding probability of only $9 \pm 2 \%$, characteristic of the nonspecific background observed in control experiments (Figure 1A). These experiments confirmed the above finding that the Aa504-610 fragments do not interact with each other. In contrast, similar binding/unbinding experiments performed with the Aa392503 fragment corresponding to the $\mathrm{N}$-terminal subdomain of the human aC-domain revealed 
homophilic interactions (Figure 1B), in agreement with the previously observed selfassociation of the N-terminal sub-domain fragments in solution. ${ }^{20,22}$ Namely, Aa392-503 coupled to a bead reacted with the pedestal-bound Aa392-503, producing rupture force spectra with three peaks centered at $16 \pm 2,32 \pm 2$, and $48 \pm 5 \mathrm{pN}$ and a cumulative binding probability of $67 \pm 8 \%$ (Figure 1B). It should be noted that the rupture force histogram produced by this interaction was very similar to that observed earlier by this method for the aC-domain-aC-domain interaction. ${ }^{24}$ The presence of three quantized peaks in the force spectrum likely reflects formation of single, double, and triple bonds and/or involvement of multiple binding sites, as was discussed before. ${ }^{24}$

Altogether, the experiments described above indicate that the C-terminal subdomain fragments do not interact with each other and do not self-associate in solution upon increasing of their concentration while the N-terminal subdomain fragments associate with each other in the same manner as the full-length aC-domains. These findings indicate that self-association of the aC-domains occurs exclusively through the homophilic interaction between their N-terminal subdomains.

\section{Testing Interactions of the $\mathrm{N}$ - and $\mathrm{C}$-Terminal Subdomains with the aC-Connector}

Since in polymeric fibrin factor XIIIa makes cross-linking between the aC-domains and aC-connectors of different fibrin molecules, ${ }^{39}$ these constituents of the aC region are both involved in the formation of fibrin aC polymers. Our previous study performed by the optical trap-based force spectroscopy revealed that the aC-domain interacts with the aCconnector. ${ }^{24}$ To identify directly which of the two subdomains of the aC-domain is involved in this interaction, we measured binding of its N-terminal subdomain (Aa392-503 fragment) and C-terminal subdomain (Aa504-610 fragment) to the aC-connector (Aa221391 fragment) using the same method. In the experiments performed, each of these subdomains was allowed to interact with the aC-connector, and their interactions were compared with that of the aC-domain (Aa392-610 fragment) under identical conditions. When the pedestal-bound aC-connector was exposed to the $\mathrm{N}$-terminal subdomain coupled to a bead, the interactions formed a decreasing spectrum of rupture forces with a cumulative binding probability of $18 \pm 3 \%$ (Figure $2 \mathrm{~A}$ ), characteristic of the nonspecific interactions with a force profile similar to that observed in the experiment presented in Figure $1 \mathrm{~A}$. In contrast, the C-terminal subdomain coupled to a bead reacted with the pedestal-bound aCconnector with a cumulative binding probability of $46 \pm 11 \%$ and a rupture force peak at 26 $\pm 2 \mathrm{pN}$ (Figure 2B). In a positive control experiment, the entire aC-domain coupled to a bead reacted with the aC-connector with a similar cumulative binding probability of $51 \pm$ $12 \%$ and a similar rupture force peak at $22 \pm 8 \mathrm{pN}$ (Figure 2C), the differences being statistically insignificant from the C-terminal subdomain. It should be noted that the binding strength for the interaction of the aC-domain with the aC-connector was very similar to that determined for this pair earlier. ${ }^{24}$ This demonstrates high reproducibility of the results obtained by this method and validates the new data. Altogether, these experiments clearly indicate that the $\mathrm{C}$-terminal subdomain of the aC-domain interacts with the aC-connector while the N-terminal subdomain does not. Furthermore, the fact that rupture forces for the interaction of the C-terminal subdomain fragment and the aC-domain fragment are similar suggests that the interaction of the aC-domain with the aC-connector occurs exclusively through its C-terminal subdomain.

Having established that the $\mathrm{C}$-terminal subdomain does not self-associate and interacts with the aC-connector and having confirmed that the N-terminal subdomains interact with each other (self-associate) to form ordered oligomers, ${ }^{20,22}$ we next focused on the latter interaction to further clarify the mechanism of aC polymer formation. 


\section{Modeling Self-Association of the N-Terminal Subdomains}

Our previous studies with the bovine Aa406-483 and human Aa425-503 fragments also revealed that the stability of the monomeric forms of these fragments is very low while that of their oligomeric forms is significantly increased. ${ }^{20,22}$ The very low stability of the monomers may be connected with their structure ${ }^{20,22}$ that includes a mixed four-stranded parallel/antiparallel $2 \uparrow 1 \downarrow 3 \downarrow 4 \uparrow \beta$-sheet in which two $\beta$-hairpins, $2 \uparrow 1 \downarrow$ and $3 \downarrow 4 \uparrow$, interact through parallel $\beta$-strands $1 \downarrow 3 \downarrow$ (Figure 3A, top). Indeed, as proposed earlier, such a topology should be unstable because the involvement of two parallel $\beta$-strands in the middle in both parallel and antiparallel hydrogen bonding may impose serious stress on the geometry of the $\beta$-sheet. ${ }^{40}$ The simplest mode of the interaction between the $\mathrm{N}$-terminal subdomain monomers to form oligomers would be through formation of hydrogen bonds between the antiparallel $\beta$-strands of the existing $\beta$-sheets arranged in a "side-by-side" manner, as shown in Figure 3A. Although our molecular modeling suggests that such interaction should not impose steric hindrance, the stability of final oligomers may be low due to preservation of the parallel/antiparallel hydrogen bonding. Thus, to explain how the structure of Aa406-483 is stabilized upon oligomerization, we hypothesized earlier that self-association of this fragment into more stable oligomers may occur by three-dimensional (3D) domain swapping mechanism, namely, by swapping the $\beta$-hairpins of the monomeric units to arrange all $\beta$-strands in antiparallel fashion. ${ }^{22}$ To test this hypothesis, we performed molecular modeling aimed to evaluate the feasibility of the hypothesized $\beta$-hairpin swapping. The modeling revealed that from a steric standpoint such swapping may indeed occur (Figure 3B). However, practical realization of such swapping would be rather difficult due to the involvement of substantial structural rearrangement upon flipping the $\beta$-hairpins (shown by curved arrows in Figure 3B, top), which may require a considerable energy toll. To address this issue, we searched for alternative models. One of the models examined is shown in Figure 3C. In this model, the swapping upon oligomerization occurs without flipping the $\beta$-hairpins but simply by displacing them aside of the existing $\beta$-sheets (shown by straight arrows in Figure 3C, top). The displaced hairpins are replaced with their counterparts from the interacting partner, allowing them to form antiparallel $\beta$-sheets. The resulting oligomer, in which $\beta$-hairpins of the neighboring $\mathrm{N}$-terminal subdomains are interlocked in a zipper-type manner, contains two $\beta$-sheets packed closely "face-to-face", resembling the topology of a $\beta$-sandwich structural motif found in many proteins. In addition to the network of hydrogen bonds typical for an antiparallel $\beta$-sheet, the stability of such oligomer should be further reinforced by hydrophobic interactions in the sandwich core.

\section{Modeling a "Disulfide Lock" in the Aa406-483 Fragment}

The $\beta$-hairpin swapping hypothesis can be further tested by "locking" the structure of the Aa 406-483 fragment in the $2 \uparrow 1 \downarrow 3 \downarrow 4 \uparrow \beta$-sheet conformation to prevent the hypothesized swapping of the $\beta$-hairpins and checking the ability of such "locked" structure to form ordered oligomers. In principle, the structure can be "locked" by the introduction of a disulfide linkage between the middle $\beta$-strands $\left(1 \downarrow{ }_{\downarrow} \downarrow\right)$, which would fasten the $\beta$-hairpins to each other. To identify pairs of residues suitable for substitution with Cys to create such a linkage (denoted "C1-C2" in Figure 4A), we performed comparative analysis of the interface between the first and second $\beta$-hairpins utilizing all available NMR structures of the bovine Aa406-483 fragment (PDB entry 2JOR). For all residues forming this interface, $\mathrm{C}_{\mathrm{a}}-\mathrm{C}_{\mathrm{a}}$ and $\mathrm{C}_{\beta}-\mathrm{C}_{\beta}$ distances, as well as orientations of side chains, were compared to determine pairs of residues with most conserved relative positions and geometries favoring the formation of disulfide bridges while causing a minimal conformational stress to the $\beta$ structure. Four pairs of residues potentially suitable for replacing with Cys to form disulfide bridges were selected: Thr428/Thr463, Lys429/Thr463, Val426/His461, and Ile427/His461. All these candidate residues were relatively distant from the existing pair of Cys residues, 
Cys423 and Cys453, thus reducing the risk of possible cross-bridging to one of these residues. They also were far enough from the tip of the second $\beta$-hairpin minimizing the interference of the newly formed disulfide bridge with the $\beta$-turn. The models were generated as described in the Experimental Procedures section; the consequences of disulfide bridge formation on the whole $\beta$-structure were analyzed with short-term molecular dynamics at a constant temperature. The superposition analysis of the original structure and its Cys derivatives suggested that the potential structural impact made by three out of four mutant candidates, namely, by Thr428Cys/Thr463Cys, Lys429Cys/Thr463Cys, and Val426Cys/His461Cys, should be accommodated rather well (Figure 4B-D). Therefore, these pairs of residues were selected for experimental mutagenesis.

\section{Preparation and Characterization of "Disulfide Locked" Mutants}

The three mutants suggested by the molecular modeling, Thr428Cys/Thr463Cys (Mut-1), Lys429Cys/Thr463Cys (Mut-2), and Val426Cys/His461 (Mut-3), have been constructed and expressed in E. coli as described in the Experimental Procedures section. Each mutant was found mainly in inclusion bodies that were dissolved in $8 \mathrm{M}$ urea and then additionally purified by size-exclusion chromatography on a Superdex 75 column equilibrated with $4 \mathrm{M}$ urea. All three mutants exhibited a single peak upon chromatography on this column in $4 \mathrm{M}$ urea (not shown) and a single band in SDS-PAGE (Figure 5A, inset). However, when these mutants were refolded by slow dialysis, as described earlier, ${ }^{16}$ and applied to a Superdex 75 column equilibrated with TBS, they exhibited complex elution profiles. Namely, the profiles of all mutants contained a minor peak, which was eluted after the free volume and represented most probably aggregated mutants, and two poorly resolved major peaks (Figure $5)$.

The fact that the position of the first of the major peaks in each mutant corresponded to that of the refolded wild-type fragment (broken line in Figure 5A) suggested that this peak corresponds to a properly folded fraction of the mutants while the second peak, which eluted later, could represent a fraction of the unfolded or improperly folded mutants. To test this suggestion, we collected two fractions corresponding to the first and second peaks of Mut-2 (Figure 5B); each fraction was then further purified to homogeneity by rechromatography, and their folding status was tested by circular dichroism (CD). The CD spectra of these two fractions were very different (Figure 5B, inset). While the spectrum of the first fraction was essentially the same as that of the wild-type fragment, the second fraction exhibited a spectrum similar to that of an unfolded protein. These results indicate that the first fraction contained the mutant whose fold was similar to that of the wild type. They also suggest that the four Cys residues in this fraction of Mut- 2 were correctly paired; i.e., they formed the expected Cys423-Cys453 and Cys429-Cys463 disulfide bonds shown in Figure 4C. To support this suggestion, the following experiments have been performed. First, treatment of the first fraction of Mut-2 with Thermo Scientific Pierce DTNB (Ellman's Reagent) ${ }^{41}$ revealed no free sulfhydryl groups, indicating that all its Cys residues were involved in disulfide bond formation. Second, when this fraction was treated with factor Xa, for which the only cleavage site in this mutant is at Arg437-Thr438 (shown by small vertical arrow in Figure 4C), SDS-PAGE analysis of the product revealed one band in nonreducing conditions and two bands with higher mobility in reducing conditions (Figure 5C, inset). This result rules out alternative Cys423-Cys429 and Cys453-Cys463 pairings, which would give two bands in both nonreducing and reducing conditions. Finally, our molecular modeling study indicates that the only possible two alternative pairings in Mut-2 (Cys423-Cys429 and Cys453-Cys463 or Cys423-Cys463 and Cys429-Cys453) would significantly affect the integrity of its first $\beta$-hairpin and make virtually impossible formation of its second $\beta$ hairpin. This should dramatically decrease the amount of $\beta$-structural elements in the mutant or even prevent their formation. Actually, the CD spectrum of the second fraction of Mut-2 
(Figure 5B, inset), which is typical for an unfolded protein, reflects most probably the presence in this fraction of the mutant with the alternatively paired Cys residues. Altogether, these data indicate that Cys residues in the first fraction of Mut-2 are paired as was expected, i.e., Cys423-Cys453 and Cys429-Cys463. Because among the three mutants the proportion of the first fraction was highest in Mut-2 (Figure 5), we focused on this mutant in the further study.

\section{Testing the Ability of the "Disulfide Locked" Mut-2 Fragment To Form Oligomers}

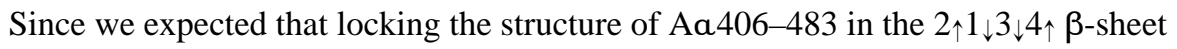
conformation would prevent the hypothesized swapping of the $\beta$-hairpins and thereby affect oligomerization, we tested the ability of Mut- 2 to form ordered oligomers. When the first fraction of this mutant was further purified to homogeneity by rechromatography, concentrated to $6 \mathrm{mg} / \mathrm{mL}$, and immediately applied to a Superdex 75 column equilibrated with TBS, practically no oligomers were observed while the wild-type Aa406-483 fragment exhibited up to $13 \%$ oligomers (Figure 6A,B, solid lines). However, after incubation for 24 $\mathrm{h}$ the same samples, Mut-2 and wild-type Aa406-483, exhibited $12 \%$ and $21 \%$ oligomers, respectively (Figure 6A,B, broken lines), suggesting that properly folded Mut-2 does form oligomers and oligomerization of both species is time-dependent. To test this suggestion, we performed a systematic study of the time dependence of oligomer formation by both species at a concentration of $3 \mathrm{mg} / \mathrm{mL}$. As shown in Figure 6B, inset, the fraction of oligomers formed by wild-type Aa406-483 reached a plateau (i.e., the oligomerization reached equilibrium) after $24 \mathrm{~h}$ while that of Mut-2 continued increasing even after $144 \mathrm{~h}$, suggesting that oligomerization of the mutant is slower than that of the wild type and the degree of oligomerization is lower.

To test if oligomerization of the mutant is reversible, as in the case with the wild-type Aa 406-483 fragment, ${ }^{22}$ the following experiments have been performed. As mentioned above, size-exclusion chromatography of Mut-2, which was concentrated to $6 \mathrm{mg} / \mathrm{mL}$ and incubated for $24 \mathrm{~h}$, revealed $12 \%$ oligomers. When this mutant fragment was then diluted to $3 \mathrm{mg} / \mathrm{mL}$, incubated for an additional $24 \mathrm{~h}$, and subjected to size-exclusion chromatography, the fraction of oligomers decreased to $6 \%$. Similar experiments were also performed in the presence of $2 \mathrm{M} \mathrm{NaCl}$, which was shown earlier to promote oligomerization of aC-domain fragments. ${ }^{20}$ Namely, size-exclusion chromatography of Mut- 2 at $3 \mathrm{mg} / \mathrm{mL}$ incubated in 2 $\mathrm{M} \mathrm{NaCl}$ for $24 \mathrm{~h}$ revealed a much higher content of oligomers, $32 \%$. When this sample was dialyzed overnight versus TBS and subjected to size-exclusion chromatography, only $6 \%$ oligomers was observed. These experiments confirmed that oligomerization of this mutant is reversible.

To gain further insight into the oligomerization process of the mutant fragment, we then tested if oligomer formation by Mut- 2 increases its thermal stability, as was observed earlier with the wild-type Aa406-483 fragment. ${ }^{22}$ When Mut-2 at $3 \mathrm{mg} / \mathrm{mL}$, at which $6 \%$ of oligomers was detected by size-exclusion chromatography of the sample incubated for $24 \mathrm{~h}$, was heated in the spectropolarimeter while the ellipticity at $227 \mathrm{~nm}$ was monitored, it exhibited a sigmoidal transition with a midpoint $\left(T_{\mathrm{m}}\right)$ at $59.3{ }^{\circ} \mathrm{C}$ (Figure $7 \mathrm{~A}$ and Table 1). Note that this $T_{\mathrm{m}}$ is much higher than that obtained for the wild-type fragment at the same concentration $\left(T_{\mathrm{m}}=27.5^{\circ} \mathrm{C}\right)$ (Figure 7B and Table 1), most probably due to the stabilizing effect of the extra disulfide in the mutant. At a concentration of $6 \mathrm{mg} / \mathrm{mL}$, at which more oligomers were detected in both Mut-2 (12\%) and the wild-type fragment (21\%), the $T_{\mathrm{m}}$ were found to be 59.7 and $34.3{ }^{\circ} \mathrm{C}$, respectively (Figure 7 and Table 1). Thus, while in the wild-type fragment further oligomerization resulted in the increase of its stability, the stability of Mut-2 remained practically unchanged. To further confirm this trend, we tested the thermal stability of Mut- 2 fragment in the presence of $2 \mathrm{M} \mathrm{NaCl}$, which, as mentioned above, promotes its oligomerization. Size-exclusion chromatography revealed a much higher 
content of oligomers at this $\mathrm{NaCl}$ concentration, $32 \%$ in Mut-2 and $69 \%$ in the wild-type fragment (Table 1). Again, while thermal stability of Aa406-483 was further increased ( $T_{\mathrm{m}}$ $=39.8^{\circ} \mathrm{C}$ ), that of Mut-2 remained practically the same $\left(T_{\mathrm{m}}=59.6^{\circ} \mathrm{C}\right.$ ) (Figure 7 and Table 1). Altogether, the experiments with Mut- 2 described above indicate that, in contrast to the wild-type Aa406-483 fragment, this mutant had a lower tendency for formation of oligomers, and its oligomerization was not accompanied by thermal stabilization of its structure.

\section{Analysis of aC Polymers from Cross-Linked Fibrin}

Most of our knowledge on the structure of $\mathrm{aC}$ polymers in fibrin came from the study of $\mathrm{aC}$ oligomers formed in solution by recombinant fragments representing the $\mathrm{aC}$ region, aCdomain, and their truncated variants (current study and refs 20,22, and 25). While the mechanisms of self-association of fibrin $\mathrm{aC}$ polymers and $\mathrm{aC}$ oligomers seem to be the same, resulting in similar extended filaments, ${ }^{25}$ the length of such filaments may differ. Theoretically, aC oligomers in solution may grow indefinitely; however, their growth may be restricted by precipitation that could occur when the oligomers reach certain length. In fact, we observed accumulation of large oligomers that precipitated upon prolonged incubation of the aC region fragment with factor XIIIa (unpublished results). In fibrin, in which aC polymers are formed on fibers, their size may be restricted by fiber length or some other factors. Thus, to further characterize aC polymers, we next evaluated their size in fibrin.

Since factor XIIIa forms nonreducible covalent bonds between monomeric units of fibrin $\mathrm{aC}$ polymers, such cross-linked polymers can be easily separated from other fibrin chains after reduction of the interchain disulfide bonds and their size can be analyzed. Such an approach has been previously used to characterize cross-linked fibrin by SDS electrophoresis in reducing conditions. ${ }^{42}$ That analysis revealed that fibrin's cross-linked a chain polymers are very heterogeneous in size; however, the size of the largest polymers was not determined since they failed to enter the gels. ${ }^{42}$ Therefore, in the present study, for the analysis of cross-linked a chain polymers, we used dynamic light scattering (DLS), which allows determining the size distribution of large particles. Furthermore, since the previously observed heterogeneity of a chain polymers ${ }^{42}$ could be connected with their incomplete cross-linking, the cross-linked fibrin used in the present study was prepared by prolonged incubation with high concentration of factor XIIIa. SDS-PAGE analysis of such fibrin, which was completely dissolved as described in the Experimental Procedures, revealed individual $\beta$ chain, cross-linked $\gamma$ chain dimer $(\gamma-\gamma)$, and high molecular mass material at the start of the gel corresponding to cross-linked $\alpha$ chain polymers (Figure 8A, inset). This analysis confirmed that the cross-linking of the $\gamma$ and a chains was complete and the interchain disulfide bonds were reduced and blocked.

The results of DLS experiments with the sample of solubilized cross-linked fibrin are presented in Figure 8A. Since DLS reports an intensity-weighted, $z$-average size distribution that is inherently sensitive to larger oligomers, ${ }^{30,43}$ these results reflect mainly scattering from cross-linked a chains whose particle size peak is centered at $\sim 200-250 \mathrm{~nm}$. Indeed, in control experiments, purified cross-linked a chain polymers exhibited a similar size distribution (Figure 8B), while a mixture of fibrinogen chains, $a, \beta$, and $\gamma$, prepared as described in Experimental Procedures, exhibited a peak centered at about $16 \mathrm{~nm}$ (Figure 8B, inset). In fact, there is a very small sharp peak on the size distribution curve of cross-linked fibrin that occurs at a similar position (shown by horizontal arrow in Figure 8A) and may reflect light scattering from non-cross-linked fibrin chains. It should be noted that the peak of cross-linked a chain polymers prepared by size-exclusion chromatography (see Experimental Procedures) was shifted toward smaller particle diameters in comparison with that of cross-linked fibrin (compare Figures $8 \mathrm{~A}$ and $8 \mathrm{~B}$ ). As such a shift may be connected 
with the loss of very large polymers upon chromatographic preparation of cross-linked a chains, we further focused on the analysis of a chain size distribution in cross-linked fibrin.

Since all DLS experiments were performed in denaturing conditions (3 M guanidine- $\mathrm{HCl}$ ), in which cross-linked a chain polymers are presumably in a random coil conformation, one can evaluate molecular masses of these polymers using the equation $R_{\mathrm{h}}=M_{\mathrm{r}}^{0.56}$. It should be noted that molecular masses $\left(M_{\mathrm{r}}\right)$ of a mixture of fibrinogen a, $\beta$, and $\gamma$ chains (14-20 $\mathrm{nm}$ particle diameter) estimated using this equation gave us a value of 33-62 $\mathrm{kDa}$ (Figure $8 \mathrm{~B}$, inset), which is in a good agreement with the real $M_{\mathrm{r}}$ of these chains. Molecular masses for the major fraction of the particles having 200-250 nm diameters (Figure 8A) were estimated at $\sim 3800-5700 \mathrm{kDa}$. Taking into account that $M_{\mathrm{r}}$ of the monomeric a chain is $64.6 \mathrm{kDa}$, these particles should consist of $\sim 60-90$ monomeric units. The correlation between larger particles and approximate number of their monomeric units $(n)$ is shown in Figure $8 \mathrm{~A}$ by vertical bars (for 350-400, 500-550, and 650-700 nm particle diameters $n$ $\sim 160-200,300-360$, and 490-550, respectively). Thus, the results of DLS experiments indicate that cross-linked aC polymers from fibrin are presented by a major fraction whose size is below 100 monomers and a fraction of larger polymers whose size is restricted by several hundred monomers.

\section{DISCUSSION}

Numerous data indicate that the $\mathrm{aC}$ domains are inactive in fibrinogen and adopt a physiologically active conformation only upon their polymerization in fibrin. 6,8,12,20,22 Our previous study suggests that formation of aC polymers in fibrin occurs through selfassociation of the aC-domains, ${ }^{20}$ which are then covalently cross-linked to the $\mathrm{aC}$ connectors of neighboring molecules by factor XIIIa. ${ }^{39}$ Thus, aC polymers are actually formed by fibrin $\mathrm{aC}$ regions that include the $\mathrm{aC}$-domains and $\mathrm{aC}$-connectors. Our recent study with the recombinant $\mathrm{aC}$ regions revealed that they self-associate in solution into highly ordered oligomers that mimic the structure and functional properties of fibrin $\mathrm{aC}$ polymers. ${ }^{25}$ In the present study, we tested the interaction between different portions of the $\mathrm{aC}$ regions, performed molecular modeling and site-directed mutagenesis of one of these portions, the N-terminal subdomain, and evaluated the size of fibrin a chain polymers to further clarify the structure of aC polymers in fibrin and molecular mechanism of their formation.

We have previously found that the $\mathrm{N}$-terminal subdomains of the aC-domains interact with each other (self-associate). ${ }^{20,22}$ This finding has been confirmed in the present study using the optical trap-based force spectroscopy. Furthermore, the results of the present study clearly indicate that their C-terminal subdomains do not interact in the same manner, leaving the interaction between the $\mathrm{N}$-terminal subdomains to be responsible for self-association of the aC-domains. We have also found that the aC-domain interacts with the aC-connector. ${ }^{24}$ The present study has revealed that this interaction occurs exclusively through the Cterminal subdomain. Thus, in fibrin these two subdomains carry out different functions: the $\mathrm{N}$-terminal subdomains are responsible for self-association of the aC-domains while the $\mathrm{C}$ terminal subdomains interact with the aC-connectors of neighboring $\mathrm{aC}$-regions, thereby contributing to self-association of the $\mathrm{aC}$ regions and further reinforcement of $\mathrm{aC}$ polymers. Taking into account that the reactive Lys and Gln residues that are cross-linked by factor XIIIa were localized in the C-terminal subdomain and the aC-connector, respectively, ${ }^{39,44,45}$ the interaction between the $\mathrm{C}$-terminal subdomains and aC-connectors may also provide the proper positioning of these residues to facilitate their covalent crosslinking by factor XIIIa. 
The data presented in this study together with our previous data on the structure and interaction of the $\mathrm{aC}$-domains and $\mathrm{aC}$ regions ${ }^{16,20,22-25,39}$ are summarized in Figure 9A, which represents schematically our current view on the structure of fibrin aC polymers and the mechanism of their formation. The model presented is based on the following findings: (i) self-association of the aC-domains occurs through the interaction between their $\mathrm{N}$ terminal subdomains (present study and refs 20 and 22); (ii) the C-terminal subdomains interact with the aC-connectors (present study); (iii) factor XIIIa covalently cross-links the C-terminal subdomains to the aC-connectors. ${ }^{39}$ Such interactions and cross-linking require antiparallel arrangement of the $\mathrm{aC}$ regions that are coming from the neighboring fibrin molecules. It should be noted that this two-dimensional model shows the established interactions between monomeric aC regions in aC polymers and their cross-linking; however, it does not reflect the exact spatial arrangement of individual subdomains in the polymers. A recent electron microscopy study of cross-linked aC region oligomers revealed that they have a width of $7.8 \pm 0.9 \mathrm{~nm}$, which is slightly less than twice the diameter of the monomeric aC region $(4.5 \pm 0.7 \mathrm{~nm}){ }^{25}$ The length of the $\mathrm{N}$-terminal subdomain $(4.5 \mathrm{~nm})^{22}$ is also comparable with the diameter of the $\mathrm{aC}$ region monomer. Thus, to make a polymer with such a width, their $\mathrm{N}$ - and C-terminal subdomains should overlap in the real 3D structure.

Our DLS experiments revealed that the size of a chain polymers from cross-linked fibrin is restricted. The major fraction of a chain polymers includes species whose size varies from about 60 to 90 monomeric units. This suggests that this many aC regions may form linear $\mathrm{aC}$ polymers in native fibrin. Since the widths of $\mathrm{aC}$ oligomers is about twice the diameter of the monomeric $\mathrm{aC}$ region, ${ }^{25}$ as mentioned above, one can estimate the length of such aC polymers at $\sim 130-200 \mathrm{~nm}$. This length is much shorter than that of individual fibrin fibers, excluding a possibility that fiber length may restrict the size of aC polymers in fibrin. At the same time, one cannot exclude the likelihood that their size may be restricted by the length of the aC-connectors that allow individual aC-domains to move from the bulk of the molecules and cluster into polymers. This is shown schematically in Figure 9B. It should be noted that the amount of the observed larger polymers consisting of up to several hundred monomers should be relatively low because light scattering from them is significantly higher than that from smaller polymers of the major fraction..$^{30,43}$ The larger polymers could be formed by cross-linking of smaller overlapped aC polymers that may come from neighboring fibers of fibrin network. This speculation is based on our previous finding that branched aC oligomer formed by the recombinant $\mathrm{aC}$ regions in solution may result from cross-linking of smaller oligomers to each other. ${ }^{25}$

As to the molecular mechanism of aC polymer formation, we have previously hypothesized that the interaction between the $\mathrm{N}$-terminal subdomains upon their self-association may involve swapping of the $\beta$-hairpins between neighboring subdomains. ${ }^{22}$ In the present study, we tested this hypothesis using molecular modeling and site-directed mutagenesis.

Molecular modeling of oligomers made of the bovine Aa406-483 fragment (N-terminal subdomain) confirmed that the hypothesized swapping can occur without any steric hindrance. It should be noted that among the two alternative models demonstrating possible $\beta$-hairpin swapping mechanism (Figure 3B,C) the model shown in Figure $3 \mathrm{C}$ is more reasonable. Although this model may not reflect the real structure of the $\mathrm{N}$-terminal subdomains in fibrin aC polymers, it demonstrates how $\beta$-hairpin swapping to form antiparallel $\beta$-sheet structures may occur without significant structural rearrangement in the monomeric units and thereby without significant energy toll. Furthermore, the width of such structure is $4.8 \mathrm{~nm}$, i.e., only slightly higher than the length of the monomeric $\mathrm{N}$-terminal subdomain. This leaves more space for accommodation of the $\mathrm{C}$-terminal subdomains in the $7.8 \mathrm{~nm}$ wide aC polymer. ${ }^{25}$ Thus, our molecular modeling study supports the hypothesized mechanism of formation of antiparallel $\beta$-sheet polymers by $\beta$-hairpin swapping. 
To test experimentally whether self-association of the N-terminal subdomains may occur through a $\beta$-hairpin swapping mechanism, we introduced an extra disulfide bond in between two neighboring $\beta$-hairpins of the Aa406-483 fragment ("disulfide lock") by site-directed mutagenesis (Figure 4). We expected that the newly formed disulfide should prevent $\beta$ hairpin swapping and thereby affect oligomerization of Aa406-483 mutant and its oligomerization-induced stability. Indeed, the experiments revealed that although this mutant had a tendency to form oligomers, the amount of such oligomers was lower than that formed by wild-type Aa406-483, and the kinetics of their formation was slower.

Furthermore, while oligomerization of wild-type Aa406-483 resulted in a significant increase of its thermal stability, no such effect was observed with the mutant. These results can be interpreted based on the models of oligomers presented in Figure 3. For all three models, oligomerization process is driven mainly by formation of hydrogen bond network between interacting antiparallel $\beta$-strands of the first and second $\beta$-hairpins of the neighboring monomeric units. Hence, oligomerization of the mutant fragment may proceed without $\beta$-hairpin swapping, resulting in parallel/antiparallel $\beta$-sheet oligomers structurally similar to those shown in Figure 3A; however, the swapping should result in antiparallel $\beta$ sheet formation, as shown in Figure 3B,C, thereby increasing the stability of the final oligomers. Therefore, in our experiments, when $\beta$-hairpin swapping in Aa406-483 mutant was prevented by the "disulfide lock", the monomers were still able to form oligomers, but this process was less efficient and the oligomers were not stabilized. Thus, the results of our experiments with Aa406-483 mutant are in agreement with the hypothesized $\beta$-hairpin swapping mechanism, albeit they do not provide direct evidence for it.

Although the three-dimensional structure of the C-terminal subdomain is not established yet, our previous study revealed that it acquires mainly regular $\beta$-conformation in oligomers formed by the aC-domains. ${ }^{20}$ Moreover, on the basis of the $\mathrm{CD}$ analysis of oligomers formed by the recombinant $\mathrm{aC}$ region, we previously hypothesized that a portion of the $\mathrm{aC}$ connectors in $\mathrm{aC}$ polymers may also acquire $\beta$-strand conformation. ${ }^{25}$ This hypothesis is in agreement with the observed significant increase of $\beta$-sheet content upon fibrin polymerization, which was suggested to occur upon interaction between the a chains forming aC regions. ${ }^{46,47}$ In this connection, it should be noted that fibronectin Fib-1 region, which includes five finger domains, interacts with bacterial fibronectin binding proteins (FnBPs) through their unstructured regions of tandem repeats, and this interaction occurs through a so-called tandem $\beta$-zipper mechanism. ${ }^{48-52}$ This mechanism includes formation of additional antiparallel $\beta$-strands by the unstructured regions of FnBPs that interact with the edges of the triple-stranded $\beta$-sheets of adjacent finger domains of Fib-1. ${ }^{48,49}$ Since the $\mathrm{C}$-terminal subdomain of the $\mathrm{aC}$-domain contains $\beta$-sheets ${ }^{20}$ and the $\mathrm{aC}$-connector of the $\mathrm{aC}$ region is unstructured ${ }^{16,23}$ and contain several tandem repeats, ${ }^{53}$ one can hypothesize that the interaction between them occurs through the same tandem $\beta$-zipper mechanism. Namely, upon this interaction, a portion of the aC-connector acquires $\beta$-strand conformation and this $\beta$-strand(s) forms hydrogen bonds with the antiparallel $\beta$-strand(s) of the $\mathrm{C}$-terminal subdomain, as shown schematically in Figure 9A, bottom.

In summary, our binding study revealed that self-association of the aC-domains into aC polymers occurs through their $\mathrm{N}$-terminal subdomains while their $\mathrm{C}$-terminal subdomains interact with the $\mathrm{aC}$ connectors, reinforcing the structure of the polymers and providing the proper orientation of their reactive residues for efficient cross-linking by factor XIIIa. The results of our molecular modeling and site-directed mutagenesis support in part the previously proposed hypothesis that interaction between the $\mathrm{N}$-terminal subdomains occurs through the $\beta$-hairpin swapping mechanism. On the basis of the results of our present and previous studies, we propose a model that describes the structure of fibrin $\mathrm{aC}$ polymers and aspects of the molecular mechanism of their formation. 


\title{
Acknowledgments
}

Funding

This work was supported by National Institutes of Health Grants HL056051 to L.M., HL030954 and HL090774 to J.W.W., and by the Intramural Research Program of the National Heart Lung, and Blood Institute to N.T.

\section{ABBREVIATIONS}

\author{
CD circular dichroism \\ DLS dynamic light scattering \\ TBS Tris-buffered saline [20 mM Tris buffer (pH 7.4) containing $150 \mathrm{mM} \mathrm{NaCl}$.
}

\section{REFERENCES}

1. Medved LV, Gorkun OV, Manyakov VF, Belitser VA. The role of fibrinogen aC-domains in the fibrin assembly process. FEBS Lett. 1985; 181:109-112. [PubMed: 3972099]

2. Gorkun OV, Veklich YI, Medved LV, Henschen AH, Weisel JW. Role of the aC domains of fibrin in clot formation. Biochemistry. 1994; 33:6986-6997. [PubMed: 8204632]

3. Collet JP, Moen JL, Veklich YI, Gorkun OV, Lord ST, Montalescot G, Weisel JW. The aC domains of fibrinogen affect the structure of the fibrin clot, its physical properties, and its susceptibility to fibrinolysis. Blood. 2005; 106:3824-3830. [PubMed: 16091450]

4. Smith KA, Adamson PJ, Pease RJ, Brown JM, Balmforth AJ, Cordell PA, Ariëns RA, Philippou H, Grant PJ. Interactions between factor XIII and the aC region of fibrinogen. Blood. 2011; 117:34603468. [PubMed: 21224475]

5. Credo RB, Curtis CG, Lorand L. a-chain domain of fibrinogen controls generation of fibrinoligase (coagulation factor XIIIa). Calcium ion regulatory aspects. Biochemistry. 1981; 20:3770-3778. [PubMed: 6115670]

6. Tsurupa G, Medved L. Identification and characterization of novel tPA- and plasminogen- binding sites within fibrin(ogen) aC-domains. Biochemistry. 2001; 40:801-808. [PubMed: 11170397]

7. Medved L, Nieuwenhuizen W. Molecular mechanisms of initiation of fibrinolysis by fibrin. Thromb. Haemost. 2003; 89:409-419. [PubMed: 12624622]

8. Tsurupa G, Yakovlev S, McKee P, Medved L. Noncovalent interaction of $a_{2}$-antiplasmin with fibrin(ogen): localization of $a_{2}$-antiplasmin-binding sites. Biochemistry. 2010; 49:7643-7651. [PubMed: 20687529]

9. Cheresh DA, Berliner SA, Vicente V, Ruggeri ZM. Recognition of distinct adhesive sites on fibrinogen by related integrins on platelets and endothelial cells. Cell. 1989; 58:945-953. [PubMed: 2673537]

10. Belkin AM, Tsurupa G, Zemskov E, Veklich Y, Weisel JW, Medved L. Transglutaminasemediated oligomerization of the fibrin(ogen) aC domains promotes integrin-dependent cell adhesion and signaling. Blood. 2005; 105:3561-3568. [PubMed: 15637140]

11. Kaijzel EL, Koolwijk P, Van Erck MGM, Van Hinsbergh VWM, De Maat MPM. Molecular weight fibrinogen variants determine angiogenesis rate in a fibrin matrix in vitro and in vivo. J. Thromb. Haemost. 2006; 4:1975-1981. [PubMed: 16961604]

12. Tsurupa G, Ho-Tin-Noe B, Angles-Cano E, Medved L. Identification and characterization of novel lysine-independent apolipoprotein(a)-binding sites in fibrin(ogen) aC-domains. J. Biol. Chem. 2003; 278:37154-37159. [PubMed: 12853452]

13. Medved L, Weisel JW. on behalf of Fibrinogen and Factor XIII Subcommittee of Scientific Standardization Committee of International Society on Thrombosis and Haemostasis. Recommendations for nomenclature on fibrinogen and fibrin. J. Thromb. Haemost. 2009; 7:355359. [PubMed: 19036059]

14. Privalov PL, Medved LV. Domains in the fibrinogen molecule. J. Mol. Biol. 1982; 159:665-683. [PubMed: 7143446] 
15. Medved LV, Gorkun OV, Privalov PL. Structural organization of C-terminal parts of fibrinogen Aa-chains. FEBS Lett. 1983; 160:291-295. [PubMed: 6224704]

16. Tsurupa G, Tsonev L, Medved L. Structural organization of the fibrin(ogen) aC-domain. Biochemistry. 2002; 41:6449-6459. [PubMed: 12009908]

17. Erickson HP, Fowler WE. Electron microscopy of fibrinogen, its plasmic fragments and small polymers. Ann. N.Y. Acad. Sci. 1983; 408:146-163. [PubMed: 6575682]

18. Weisel JW, Stauffacher CV, Bullitt E, Cohen C. A model for fibrinogen: domains and sequence. Science. 1985; 230:1388-1391. [PubMed: 4071058]

19. Veklich YI, Gorkun OV, Medved LV, Nieuwenhuizen W, Weisel JW. Carboxyl-terminal portions of the a chains of fibrinogen and fibrin. Localization by electron microscopy and the effects of isolated aC fragments on polymerization. J. Biol. Chem. 1993; 268:13577-13585. [PubMed: 8514790]

20. Tsurupa G, Hantgan RR, Burton RA, Pechik I, Tjandra N, Medved L. Structure, stability, and interaction of the fibrin(ogen) aC-domains. Biochemistry. 2009; 48:12191-12201. [PubMed: 19928926]

21. Burton RA, Tsurupa G, Medved L, Tjandra N. Identification of an ordered compact structure within the recombinant bovine fibrinogen aC-domain fragment by NMR. Biochemistry. 2006; 45:2257-2266. [PubMed: 16475814]

22. Burton R, Tsurupa G, Hantgan RR, Tjandra N, Medved L. NMR solution structure, stability, and interaction of the recombinant bovine fibrinogen aC-domain fragment. Biochemistry. 2007; 46:8550-8560. [PubMed: 17590019]

23. Weisel JW, Medved L. The structure and function of the aC domains of fibrinogen. Ann. N.Y. Acad. Sci. 2001; 936:312-327. [PubMed: 11460487]

24. Litvinov RI, Yakovlev SV, Tsurupa G, Gorkun OV, Medved L, Weisel J. Direct evidence for specific interactions of the fibrinogen aC-domains with the central $\mathrm{E}$ region and with each other. Biochemistry. 2007; 46:9133-9142. [PubMed: 17630702]

25. Tsurupa G, Mahid A, Veklich Y, Weisel JW, Medved L. Structure, stability, and interaction of fibrin aC-domain polymers. Biochemistry. 2011; 50:8028-8037. [PubMed: 21806028]

26. Gasteiger, E.; Hoogland, C.; Gattiker, A.; Duvaud, S.; Wilkins, MR.; Appel, RD.; Bairoch, A. Protein Identification and Analysis Tools on the ExPASy Server. In: Walker, JM., editor. The Proteomics Protocols Handbook. Totowa, NJ: Humana Press; 2005. p. 571-607.

27. Litvinov RI, Shuman H, Bennett JS, Weisel JW. Binding strength and activation state of single fibrinogen-integrin pairs on living cells. Proc. Natl. Acad. Sci. U. S. A. 2002; 99:7426-7431. [PubMed: 12032299]

28. Litvinov RI, Bennett JS, Weisel JW, Shuman H. Multi-step fibrinogen binding to the integrin aIIb $\beta 3$ detected using force spectroscopy. Biophys. J. 2005; 89:2824-2834. [PubMed: 16040750]

29. Litvinov RI, Gorkun OV, Owen SF, Shuman H, Weisel JW. Polymerization of fibrin: specificity, strength, and stability of knob-hole interactions studied at the single-molecule level. Blood. 2005; 106:2944-2951. [PubMed: 15998829]

30. Pecora, R. Quasi-elastic light scattering of macromolecules and particles in solution and suspension. In: Dahneke, BE., editor. Measurement of Suspended Particles by Quasi-Elastic Light Scattering. 1st ed.. New York: John Wiley; 1983. p. 3-30.

31. Hantgan RR, Stahle MC, Connor JH, Lyles DS, Horita DA, Rocco M, Nagaswami C, Weisel JW, McLane MA. The disintegrin Echistatin stabilizes integrin aIIb $\beta 3$ 's open conformation and promotes its oligomerization. J. Mol. Biol. 2004; 342:1625-1636. [PubMed: 15364586]

32. Provencher SW. CONTIN: A general purpose constrained regularization program for inverting noisy linear algebraic and integral equations. Comput. Phys. Commun. 1982; 27:229-242.

33. Kawahara K, Tanford C. Viscosity and density of aqueous solutions of urea and guanidine hydrochloride. J. Biol. Chem. 1966; 241:3228-3232. [PubMed: 5912116]

34. Cantor, CR.; Schimmel, PR. Biophysical Chemistry. Part II: Techniques for the Study of Biological Structure and Function. San Francisco: W.H. Freeman and Company; 1980. Size and shape of macromolecules; p. 539-590. 
35. Wilkins DK, Grimshaw SB, Receveur V, Dobson CM, Jones JA, Smith LJ. Hydrodynamic radii of native and denatured proteins measured by pulse field gradient NMR techniques. Biochemistry. 1999; 38:16424-16431. [PubMed: 10600103]

36. Stafford, WF, III. Analytical Ultracentrifugation Workshop: Theory and Practice. Storrs, CT: National Analytical Ultracentrifugation Facility, Biotechnology Center, University of Connecticut; 1996. Hydrodynamic parameters - some rules of thumb; p. 20

37. Brunger AT, Adams PD, Clore GM, DeLano WL, Gros P, Grosse-Kunstleve RW, Jiang J-S, Kuszewski J, Nigels M, Pannu NS, Read RJ, Rice LM, Simonson T, Warren GL. Crystallography and NMR system (CNS): A new software system for macromolecular structure determination. Acta Crystallogr. 1998; D54:905-921.

38. Brunger, AT. A System for X-ray Crystallography and NMR. New Haven, CT: Yale University Press; 1992. X-PLOR.

39. Matsuka YV, Medved LV, Migliorini MM, Ingham KC. Factor XIIIa-catalyzed cross-linking of recombinant aC fragments of human fibrinogen. Biochemistry. 1996; 35:5810-5816. [PubMed: 8639541]

40. Zhang C, Kim SH. The anatomy of protein $\beta$-sheet topology. J. Mol. Bol. 2000; 299:1075-1089.

41. Ellman GL. Tissue sulfhydryl groups. Arch. Biochem. Biophys. 1959; 82:70-77. [PubMed: 13650640]

42. Francis CW, Marder VJ. Rapid formation of large molecular weight a-polymers in cross-linked fibrin induced by high factor XIII concentrations. Role of platelet factor XIII. J. Clin. Invest. 1987; 80:1459-1465. [PubMed: 3680507]

43. Knoll D, Hantgan R, Williams J, McDonagh J, Hermans J. Characterization of soluble polymerized fibrin formed in the presence of excess fibrinogen fragment D. Biochemistry. 1984; 23:37083715. [PubMed: 6236846]

44. Cottrell BA, Strong DD, Watt KW, Doolittle RF. Amino acid sequence studies on the a of human fibrinogen. Exact location of cross-linking acceptor sites. Biochemistry. 1979; 18:5405-5410. [PubMed: 518845]

45. Corcoran DH, Ferguson EW, Fretto LJ, McKee PA. Localization of a cross-link donor site in the alpha-chain of human fibrin. Thromb. Res. 1980; 19:883-888. [PubMed: 6781100]

46. Marx J, Hudry-Clergeon G, Capet-Antonini F, Bernard L. Laser Raman spectroscopy study of bovine fibrinogen and fibrin. Biochim. Biophys. Acta. 1979; 578:107-115. [PubMed: 454660]

47. Hudry-Clergeon G, Freyssinet J-M. Orientation of fibrin in strong magnetic fields. Ann. N.Y. Acad. Sci. 1983; 408:380-387. [PubMed: 6575695]

48. Schwarz-Linek U, Werner JM, Pickford AR, Gurusiddappa S, Kim JH, Pilka ES, Briggs JA, Gough TS, Höök M, Campbell ID, Potts JR. Pathogenic bacteria attach to human fibronectin through a tandem $\beta$-zipper. Nature. 2003; 423:171-181.

49. Schwarz-Linek U, Höök M, Potts JR. The molecular basis of fibronectin-mediated bacterial adherence to host cells. Mol. Microbiol. 2004; 52:631-641. [PubMed: 15101971]

50. Schwarz-Linek U, Höök M, Potts JR. Fibronectin-binding proteins of gram-positive cocci. Microbes Infect. 2006; 8:2291-2298. [PubMed: 16782385]

51. Raibaud S, Schwarz-Linek U, Kim JH, Jenkins HT, Baines ER, Gurusiddappa S, Höök M, Potts JR. Borrelia burgdorferi binds fibronectin through a tandem $\beta$-zipper, a common mechanism of fibronectin binding in Staphylococci, Streptococci, and Spirochetes. J. Biol. Chem. 2005; 280:18803-18809. [PubMed: 15737988]

52. Bingham RJ, Rudino-Pinera E, Meenan NAG, Schwarz-Linek U, Turkenburg JP, Hook M, Garman EF, Potts JR. Crystal structure of fibronectin-binding sites from Staphylococcus aureus FnBPA in complex with fibronectin domains. Proc. Natl. Acad. Sci. U. S. A. 2008; 105:1225412258. [PubMed: 18713862]

53. Doolittle RF, Watt KWK, Cottrell BA, Strong DD, Riley M. The amino acid sequence of the achain of human fibrinogen. Nature. 1979; 280:464-468. [PubMed: 460425]

54. DeLano, WL. PyMol. San Carlos, CA: DeLano Scientific; 2000. 


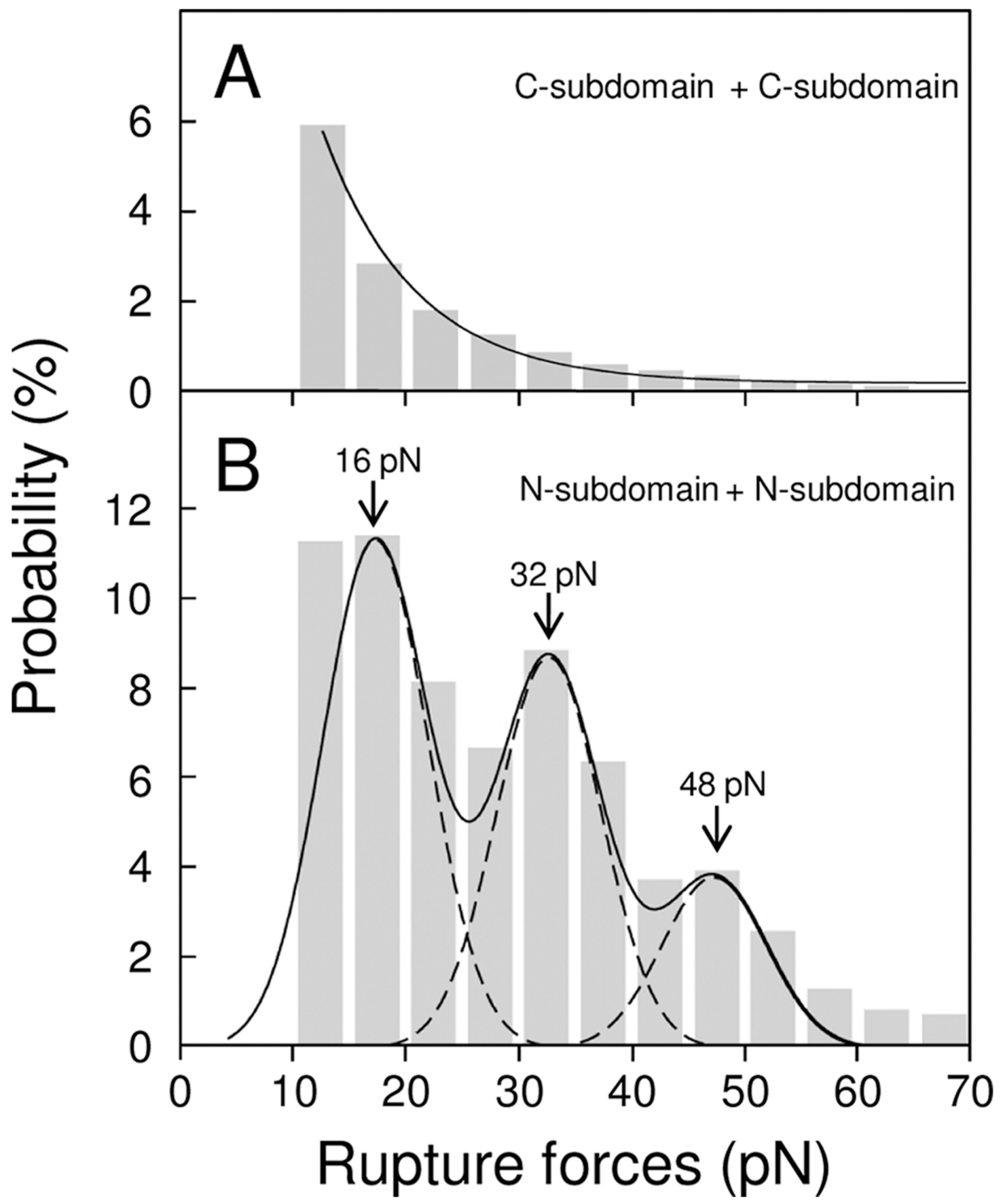

Figure 1.

Panel of rupture force histograms demonstrating homophilic interactions or lack thereof of the $\mathrm{N}$ - and C-terminal subdomains of the aC-domain. (A) Exponentially decreasing rupture forces, indistinguishable from the nonspecific background, demonstrating lack of specific interactions between two surfaces coated with the C-terminal subdomains (human Aa504610 fragments). (B) Pronounced interactions between two surfaces coated with the Nterminal subdomain (human Aa392-503 fragment), reflecting the multimodal rupture force distribution; the solid and dashed lines show the fitting with Gaussian curves to determine position of each peak. Each histogram is based on the data collected from at least 10 beadpedestal pairs, representing $10^{3}-10^{4}$ individual measurements. The number of events in each 
bin was plotted against the average force for that bin after normalizing for the total number of interaction cycles. 


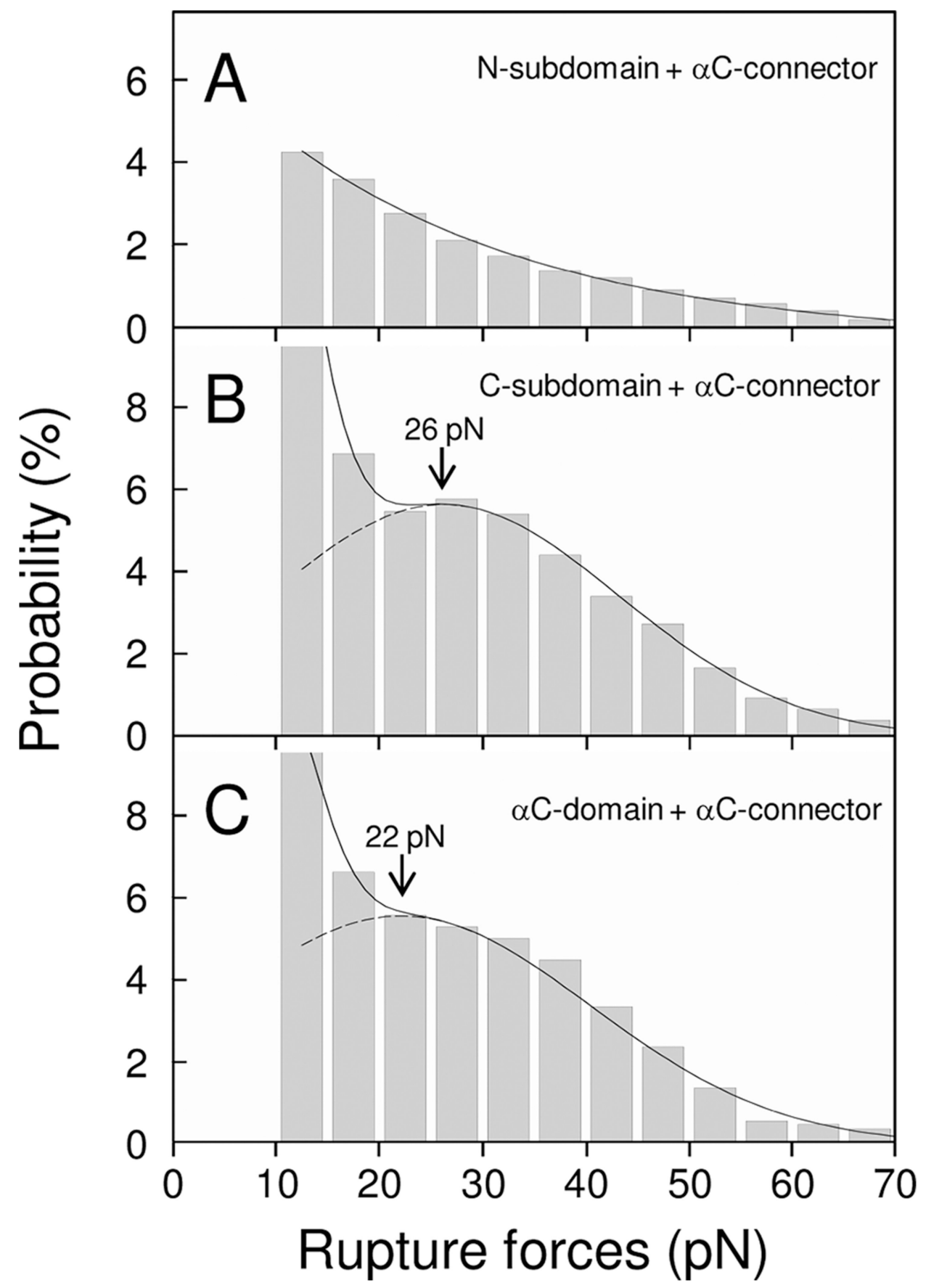

Figure 2.

Panel of rupture force histograms demonstrating interactions of the aC-domain and its $\mathrm{N}$ and $\mathrm{C}$-terminal subdomains with the aC-connector. Panels A, B, and $\mathrm{C}$ show interaction of the pedestal-bound aC-connector (human Aa221-391 fragment) with the bead-bound Nterminal subdomain (human Aa392-503 fragment), C-terminal subdomain (human Aa504610 fragment), and aC-domain (human Aa392-610 fragment), respectively. The solid lines in all panels show the fitting with exponential/Gaussian and the dashed lines in panels B and $\mathrm{C}$ show the fitting with Gaussian to determine the position of each peak. Each histogram is based on the data collected from at least 10 bead-pedestal pairs, representing $10^{3}-10^{4}$ 
individual measurements. The number of events in each bin was plotted against the average force for that bin after normalizing for the total number of interaction cycles. 


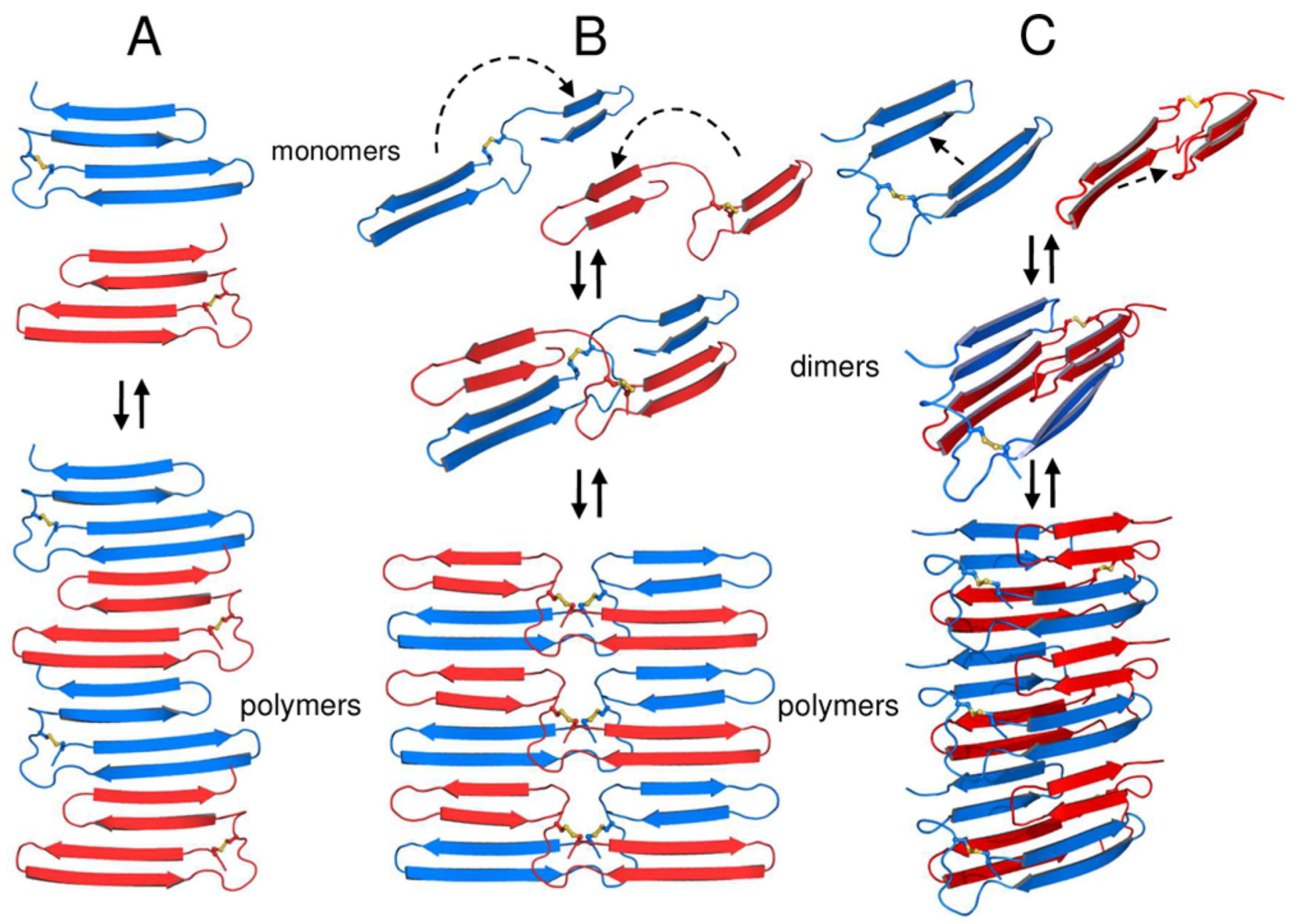

Figure 3.

Ribbon diagrams representing the results of molecular modeling of oligomer formation by the Aa406-483 fragment ( $\mathrm{N}$-terminal subdomain of the bovine aC-domain). Individual monomeric units are shown in red and blue for clarity; their $\beta$-strands are presented by wide arrows. Panel A demonstrates a "side-by-side" arrangement of the interacting units, panel B illustrates $\beta$-hairpin swapping mechanism suggested earlier, ${ }^{22}$ and panel $C$ represents an alternative mode leading to the formation of a $\beta$-sandwich-like structure (see text). Curved and straight dashed arrows at the top show respectively flipping and displacement of individual $\beta$-hairpins in the monomers to form the hypothesized dimers and polymers; double thin arrows demonstrate reversibility of the process. The molecular modeling was performed as described in the Experimental Procedures section; all ribbon diagrams were prepared with PyMOL. ${ }^{54}$ 

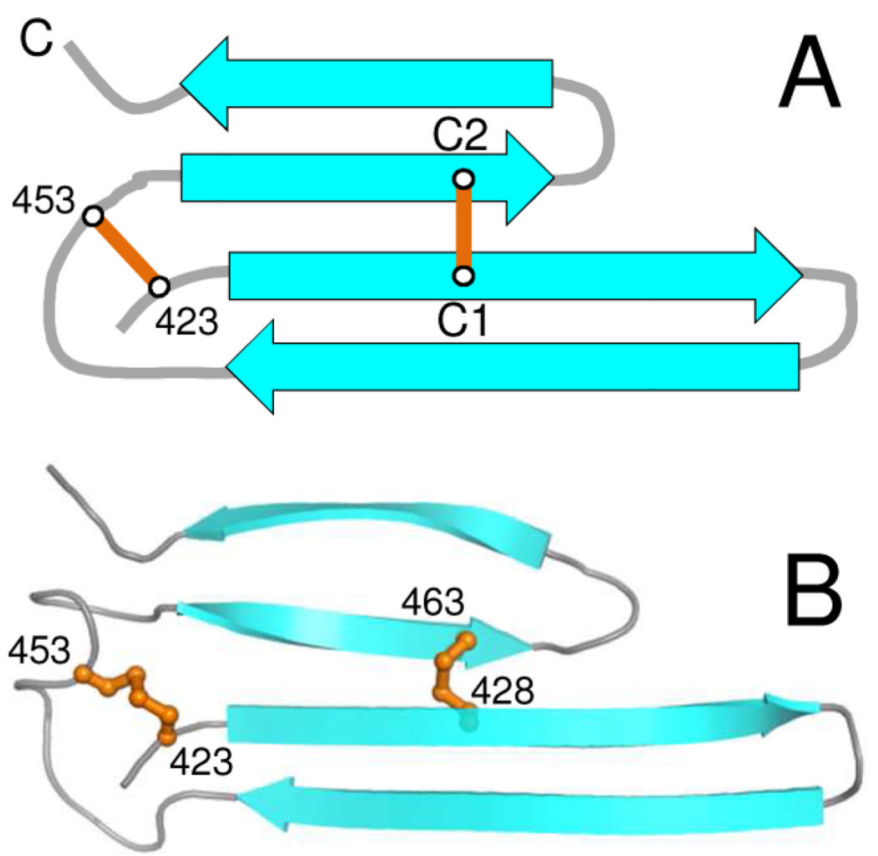

Mut-1

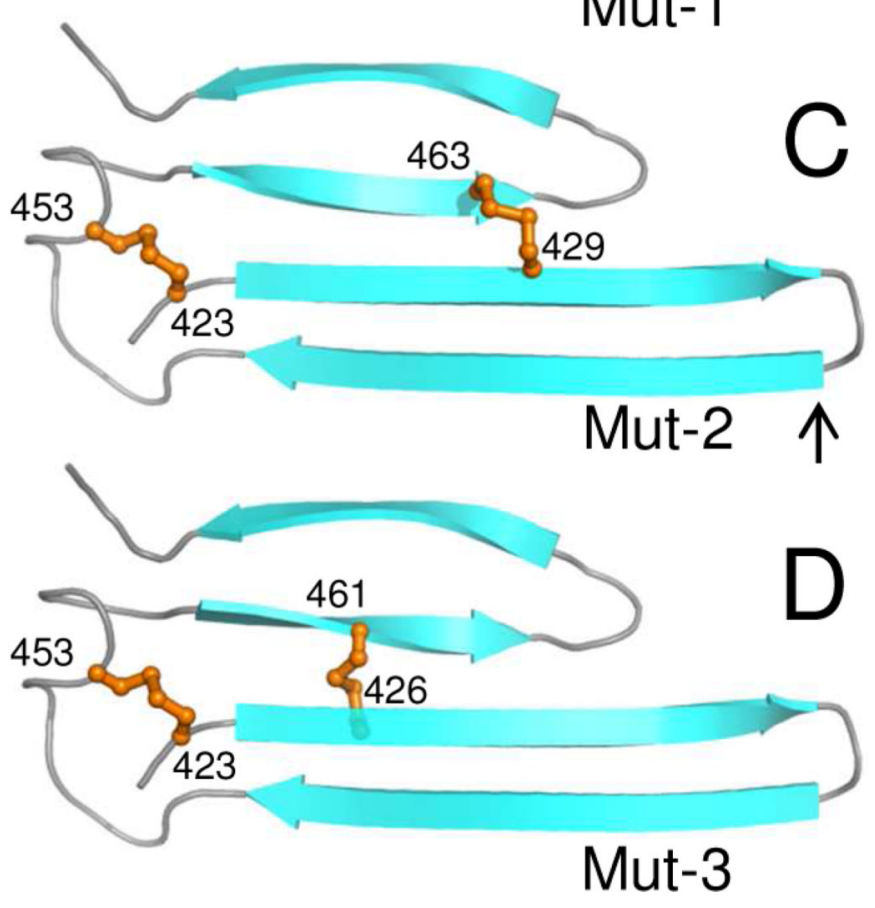

Figure 4.

Schematic representation of the bovine Aa406-483 fragment (A) and ribbon diagrams representing structural models of this fragment containing the proposed Cys mutations (BD). Panel A depicts a diagram showing the existing Cys423-Cys453 disulfide bond and a disulfide bond to be introduced between the two parallel $\beta$-strands (denoted "C1-C2"); the disulfides and $\beta$-strands are presented by orange sticks and arrows, respectively. Ribbon diagrams of the proposed mutants, Thr428Cys/Thr463Cys (Mut-1), Lys429Cys/Thr463Cys (Mut-2), and Val426Cys/His461 (Mut-3), are shown in panels B, C, and D, respectively; the existing disulfide and the disulfides suggested by the molecular modeling (Cys428-Cys463, Cys429-Cys463, and Cys426-Cys461) are shown by orange sticks. Small vertical arrow in 
panel $\mathrm{C}$ indicates cleavage site for factor Xa (see text). The molecular modeling of the mutants was performed as described in the Experimental Procedures section; the ribbon diagrams were prepared with PyMOL. ${ }^{54}$ 


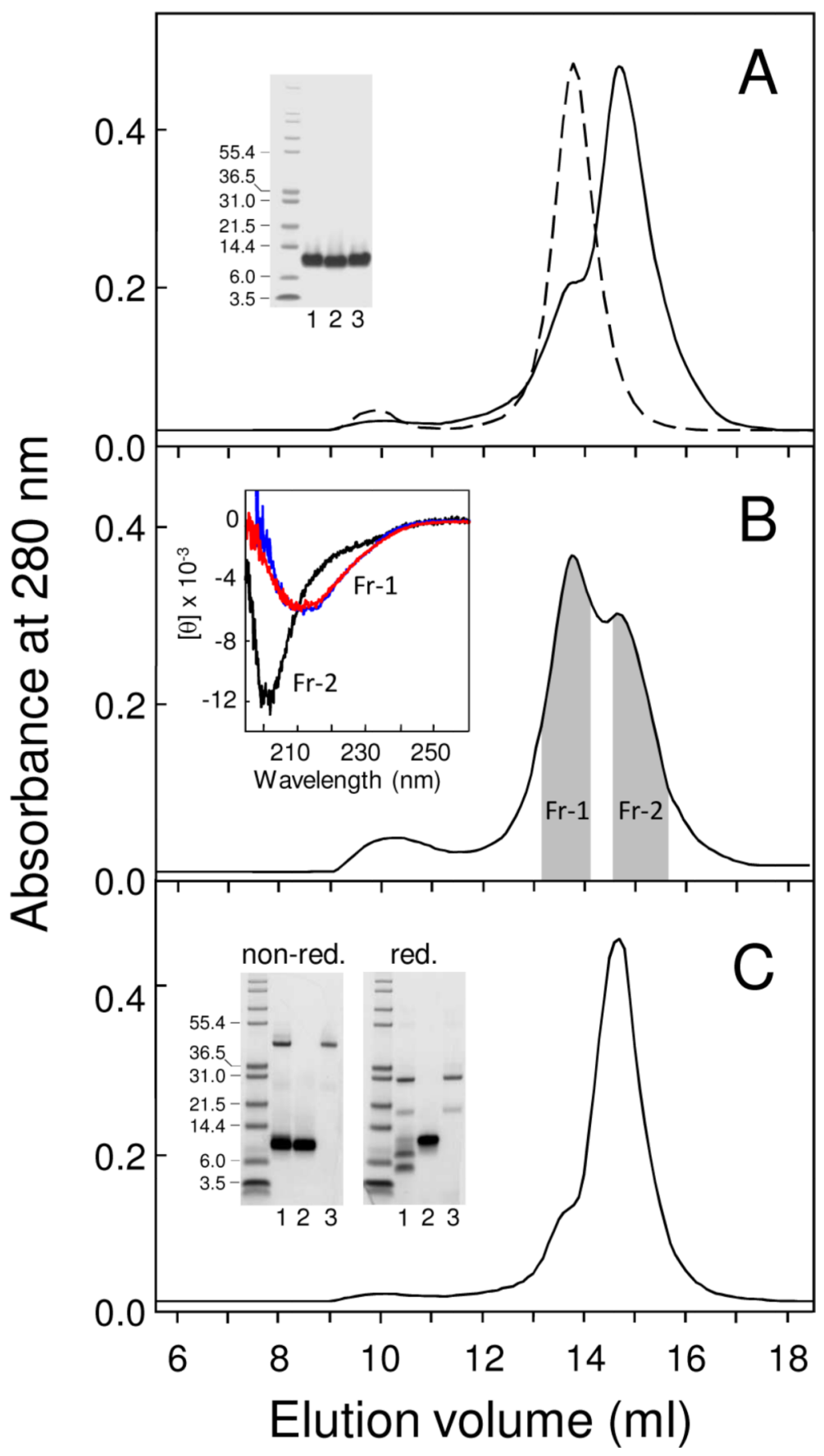

Figure 5.

Size-exclusion chromatography of the Aa406-483 mutants, Mut-1 (A), Mut-2 (B), and Mut-3 (C) on a Superdex 75 column. The chromatography profiles of the mutants in all panels are shown by solid lines; the profile of the wild-type bovine Aa406-483 fragment is shown by broken line in panel A. All experiments were performed in TBS at $4{ }^{\circ} \mathrm{C}$. Inset in panel A shows analysis of Mut-1 (lane 1), Mut-2 (lane 2), and Mut-3 (lane 3) by SDSpolyacrylamide gel electrophoresis; the left outer lane contains Mark 12 protein markers of the indicated molecular masses (in $\mathrm{kDa}$ ). The shaded areas in panel $\mathrm{B}$ indicate two fractions of the Mut-2 fragment, Fr-1 and Fr-2, collected for further analysis (see text). CD spectra of Fr-1 (red) and Fr-2 (black) are shown in panel B, inset; CD spectrum of the wild-type 
Aa406-483 fragment (blue), which essentially coincides with that of the first fraction of Mut-2, is shown for comparison. All spectra were obtained in TBS at $4{ }^{\circ} \mathrm{C}$. Inset in panel C shows the results of SDS-PAGE analysis of the first fraction of Mut-2 (Fr-1) treated with factor Xa in nonreducing (left gel) and reducing (right gel) conditions (lines 1); lines 2 and 3 show nontreated Mut-2 Fr-1 and factor Xa, respectively, and the left outer lanes contain Mark 12 protein markers of the indicated molecular masses. 


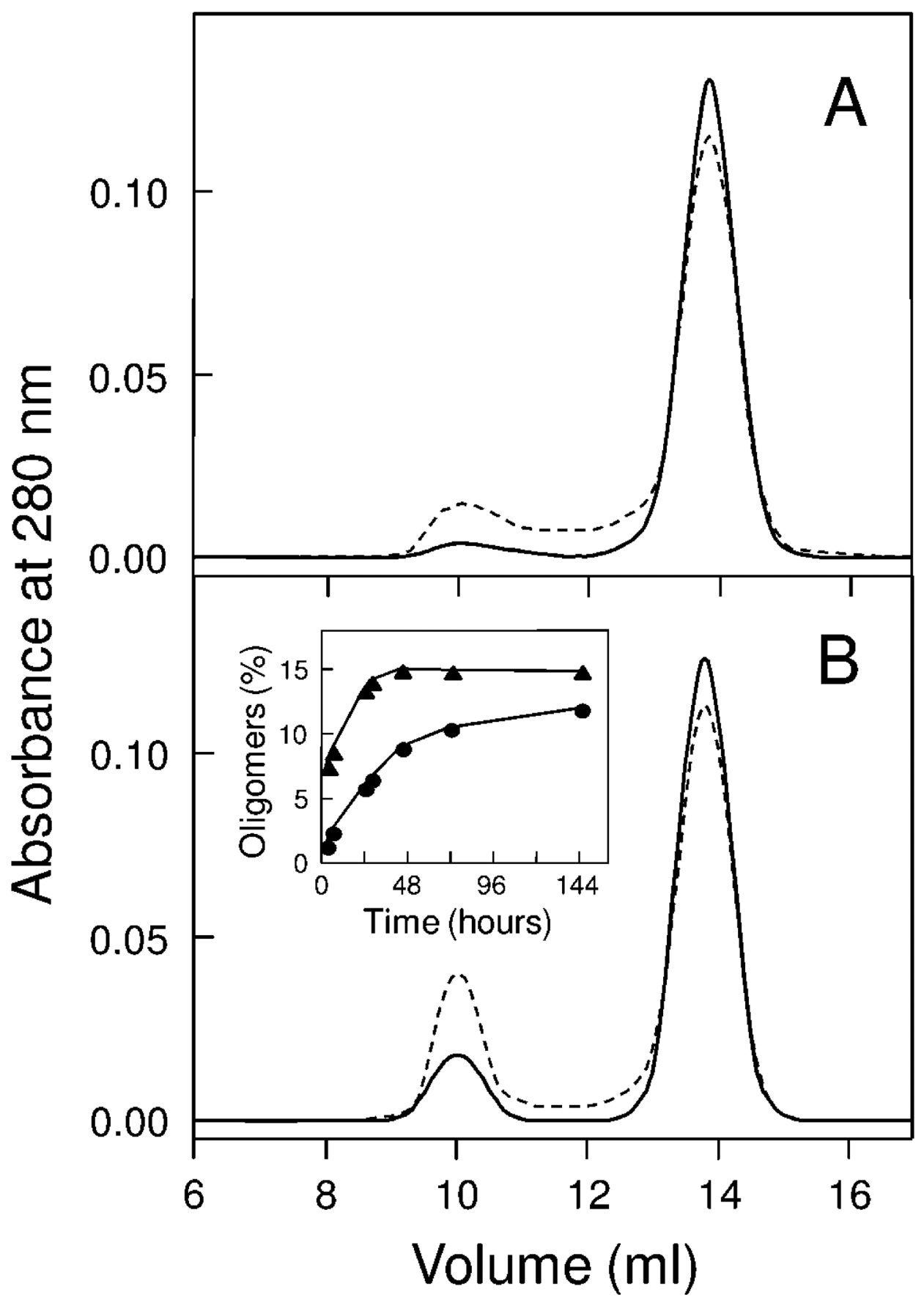

Figure 6.

Size-exclusion chromatography profiles of the purified first fraction of the Mut-2 fragment (A) and the wild-type bovine Aa406-483 fragment (B), both at $6 \mathrm{mg} / \mathrm{mL}$, applied to the column immediately after concentration (solid lines) and after incubation for $24 \mathrm{~h}$ (broken lines). Inset in panel B shows time course of oligomer formation by the wild-type Aa406483 fragment (triangles) and the first fraction of Mut-2 fragment (circles), both at $3 \mathrm{mg} / \mathrm{mL}$. All experiments were performed in TBS at $4{ }^{\circ} \mathrm{C}$. 


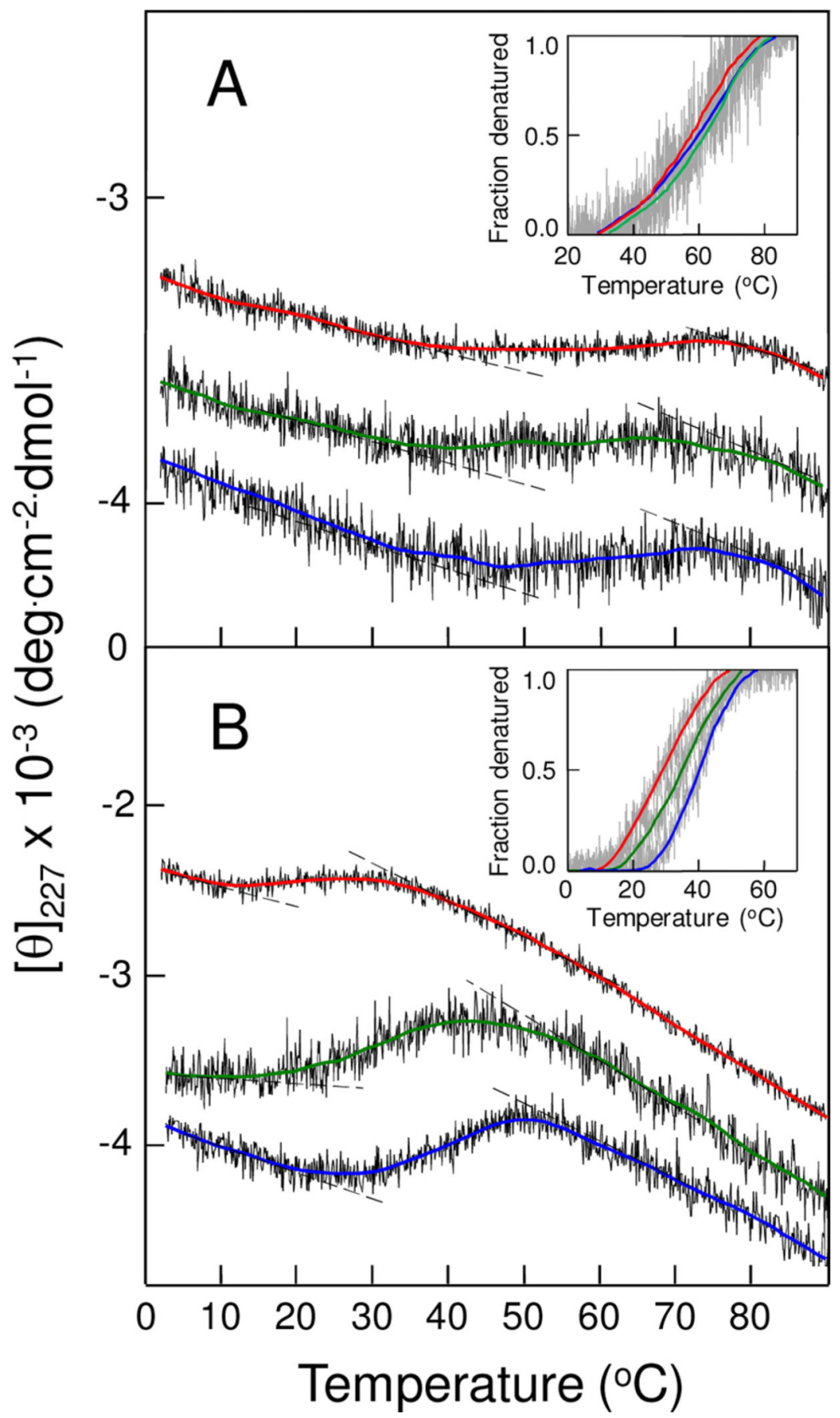

Figure 7.

CD-detected thermal unfolding of the purified first fraction of Mut-2 fragment (A) and the wild-type bovine Aa406-483 fragment (B). The unfolding experiments with both fragments were performed in $20 \mathrm{mM}$ Tris buffer $(\mathrm{pH} 7.4)$ containing $0.15 \mathrm{M} \mathrm{NaCl}$ at fragment concentrations of 3 and $6 \mathrm{mg} / \mathrm{mL}$ (red and green curves, respectively) and in $20 \mathrm{mM}$ Tris buffer (pH 7.4) containing $2 \mathrm{M} \mathrm{NaCl}$ at fragment concentrations of $3 \mathrm{mg} / \mathrm{mL}$ (blue curve). The unfolding curves have been arbitrarily shifted along the vertical axis to improve visibility and smoothed (colored curved lines) to reduce the noise. The dashed straight lines represent the results of fitting pre- and post-transition data; they provide the basis for 
estimating the fraction denatured at $3 \mathrm{mg} / \mathrm{mL}$ (red curve), $6 \mathrm{mg} / \mathrm{mL}$ (green curve), and $3 \mathrm{mg}$ / $\mathrm{mL}$ in $2 \mathrm{M} \mathrm{NaCl}$ (blue curve) presented in the insets of both panels. 


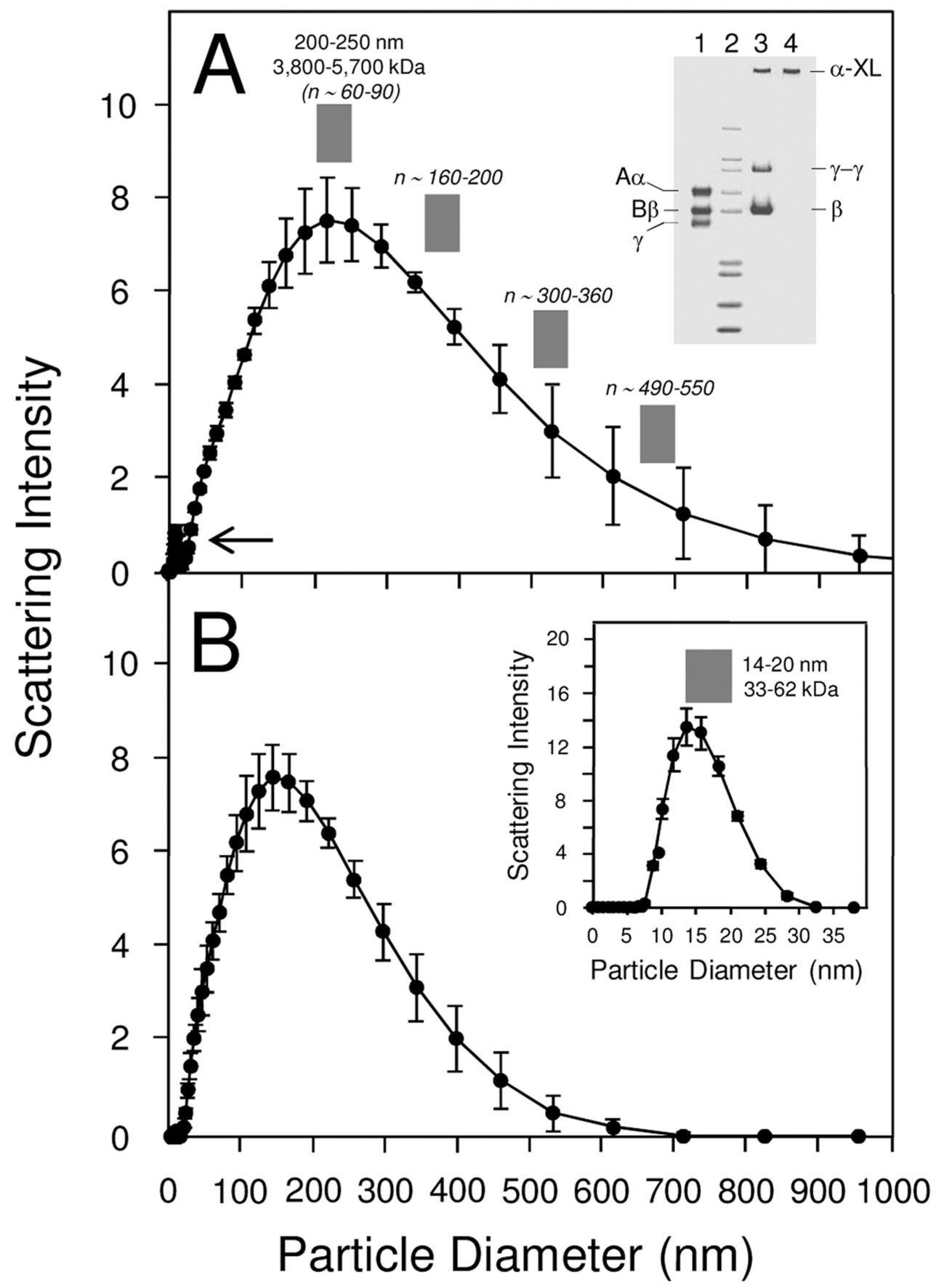

Figure 8.

Size distributions of solubilized cross-linked fibrin chains (A) and cross-linked fibrin a chain polymers (B) obtained by dynamic light scattering. Symbols denote the average and standard deviation of data obtained in triplicate determinations of the intensity autocorrelation function for each sample, analyzed by the CONTIN algorithm to obtain the $z$-average size distributions ${ }^{32}$ with correction for the increased viscosity $(\sim 18 \%)$ in $3 \mathrm{M}$ guanidine- $\mathrm{HCl} .{ }^{33,34}$ Vertical bars in panel A show correlation between the size of a chain polymers and approximate number of their monomeric units $(n)$. Inset in panel A shows SDS-PAGE analysis of samples containing reduced/blocked fibrinogen containing Aa, B $\beta$, and $\gamma$ chains (lane 1), reduced/blocked cross-linked fibrin containing $\beta$ and $\gamma-\gamma$ chains and 
cross-linked (XL) a chain polymers (lane 3 ), and purified cross-linked a chain polymers (lane 4); Mark 12 molecular mass markers (14.4, 21.5, 31.0, 36.5, 55.4, 66.3, 97.4, 116.3, and $220.0 \mathrm{kDa}$ ) are shown in lane 2. Inset in panel B shows size distributions of reduced/ blocked fibrinogen chains. 

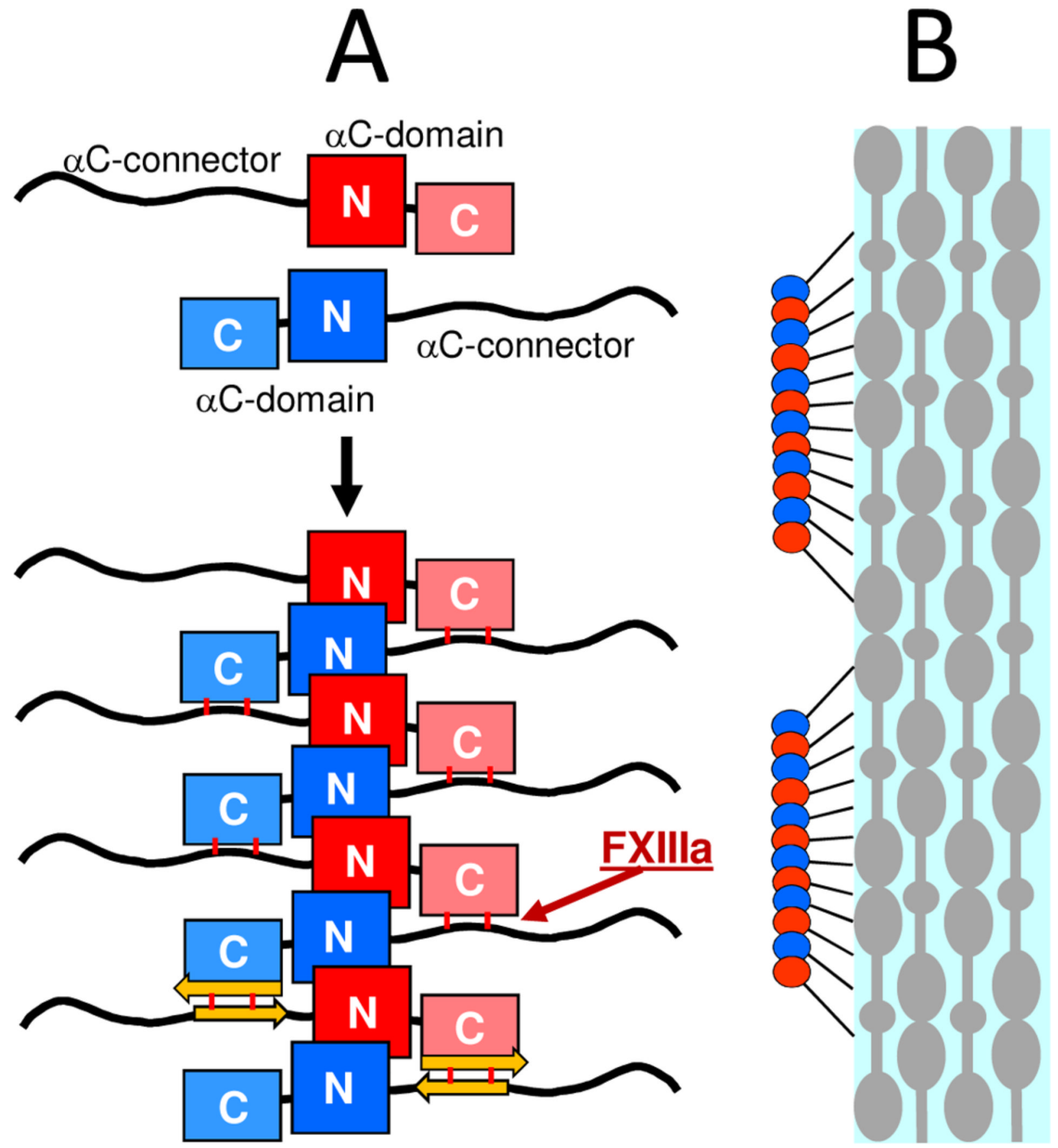

Figure 9.

Schematic presentation of fibrin aC polymers. Panel A shows monomeric aC regions (top), whose interaction through the $\mathrm{N}$-terminal subdomains (denoted by "N") results in formation of a polymer; the C-terminal subdomains (denoted by "C") interact with the aC-connectors and get cross-linked by factor XIIIa (cross-linking is shown by vertical red bars), further reinforcing the structure of the polymer. The identical monomeric aC-domains coming from opposite directions are shown in different colors, red and blue, for clarity. The two pairs of antiparallel $\beta$-strands (orange arrows at the bottom) show schematically how the C-terminal subdomains and aC-connectors may interact through tandem $\beta$-zipper mechanism (see text). Panel B represents schematically aC polymers, in which the aC-domains (blue and red 
balls) are attached to the surface of fibrin fiber through the aC-connectors (black lines), whose length may restrict the length of the polymers (see text); formation of such polymers within the fiber is also likely. 


\section{Table 1}

CD-Detected Thermal Stability of the Wild-Type Bovine Aa406-483 Fragment and the First Fraction (Fr-1) of Its Lys429Cys/Thr463Cys Mutant (Mut-2) and Their Aggregation State Determined by Size-Exclusion Chromatography

\begin{tabular}{lclrl}
\hline & \multicolumn{2}{c}{ conditions } & & \\
\cline { 2 - 4 } fragments $^{\boldsymbol{a}}{ }^{\boldsymbol{4}}$ & $\begin{array}{c}\text { fragment conc } \\
(\mathbf{m g} / \mathbf{m L})\end{array}$ & $\begin{array}{l}\mathbf{N a C l} \\
\mathbf{c o n c}(\mathbf{M})\end{array}$ & $\begin{array}{r}\text { oligomers } \\
(\boldsymbol{\%})^{\boldsymbol{b}}\end{array}$ & $\boldsymbol{T}_{\mathbf{m}}\left({ }^{\circ} \mathbf{C}\right)^{\boldsymbol{b}}$ \\
\hline Mut-2 (Fr-1) & 3.0 & 0.15 & $5.9 \pm 0.6$ & $59.3 \pm 0.6$ \\
& 6.0 & 0.15 & $12.4 \pm 1.8$ & $59.7 \pm 0.4$ \\
& 3.0 & 2.0 & $31.7 \pm 1.7$ & $59.6 \pm 0.5$ \\
Aa406-483 & 3.0 & 0.15 & $13.7 \pm 0.4$ & $27.5 \pm 0.5$ \\
& 6.0 & 0.15 & $20.9 \pm 0.4$ & $34.3 \pm 0.6$ \\
& 3.0 & 2.0 & $69.2 \pm 1.3$ & $39.8 \pm 0.4$
\end{tabular}

${ }^{a}$ All fragments were in $20 \mathrm{mM}$ Tris buffer ( $\mathrm{pH} 7.4$ ) containing the indicated concentrations of $\mathrm{NaCl}$; they were incubated for $24 \mathrm{~h}$ before sizeexclusion chromatography and CD measurements.

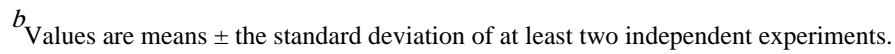

